\title{
Ibrutinib treatment improves T cell number and function in CLL patients
}

\author{
Meixiao Long,,$^{1,2}$ Kyle Beckwith, ${ }^{1,2,3}$ Priscilla Do, ${ }^{1,2,3}$ Bethany L. Mundy, ${ }^{1,2}$ Amber Cordon, ${ }^{1,2}$ Amy M. Lehman, ${ }^{2,4}$ Kami J. Maddocks, ${ }^{1,2}$ \\ Carolyn Cheney, ${ }^{2}$ Jeffrey A. Jones, ${ }^{1,2}$ Joseph M. Flynn, ${ }^{1}$ Leslie A. Andritsos, ${ }^{1,2}$ Farrukh Awan, ${ }^{1,2}$ Joseph A. Fraietta, ${ }^{5}$ Carl H. June, ${ }^{5}$ \\ Marcela V. Maus, ${ }^{6}$ Jennifer A. Woyach, ${ }^{1,2}$ Michael A. Caligiuri, ${ }^{1,2}$ Amy J. Johnson, ${ }^{1,2}$ Natarajan Muthusamy, ${ }^{1,2}$ and John C. Byrd ${ }^{1,2}$ \\ 'Division of Hematology, Department of Internal Medicine, The Ohio State University, Columbus, Ohio, USA. ${ }^{\top}$ The Ohio State University Comprehensive Cancer Center, Columbus, Ohio, USA. ${ }^{3}$ Biomedical \\ Sciences Graduate Program, The Ohio State University, ${ }^{4}$ Center for Biostatistics, The Ohio State University, Columbus, Ohio, USA. ${ }^{5}$ Pathology and Laboratory Medicine, University of Pennsylvania, \\ Philadelphia, Pennsylvania, USA. ${ }^{6}$ Massachusetts General Hospital Cancer Center, Harvard Medical School, Boston, Massachusetts, USA.
}

\begin{abstract}
BACKCROUND. Ibrutinib has been shown to have immunomodulatory effects by inhibiting Bruton's tyrosine kinase (BTK) and IL-2-inducible T cell kinase (ITK). The relative importance of inhibiting these 2 kinases has not been examined despite its relevance to immune-based therapies.
\end{abstract}

METHODS. Peripheral blood mononuclear cells from chronic lymphocytic leukemia (CLL) patients on clinical trials of ibrutinib (BTK/ITK inhibitor; $\boldsymbol{n}=19$ ) or acalabrutinib (selective BTK inhibitor; $\boldsymbol{n}=13$ ) were collected serially. T cell phenotype, immune function, and CLL cell immunosuppressive capacity were evaluated.

RESULTS. Ibrutinib markedly increased CD4 ${ }^{+}$and CD8 ${ }^{+} \mathrm{T}$ cell numbers in CLL patients. This effect was more prominent in effector/effector memory subsets and was not observed with acalabrutinib. Ex vivo studies demonstrated that this may be due to diminished activation-induced cell death through ITK inhibition. PD-1 and CTLA-4 expression was significantly markedly reduced in T cells by both agents. While the number of Treg cells remained unchanged, the ratio of these to conventional $C D 4^{+} T$ cells was reduced with ibrutinib, but not acalabrutinib. Both agents reduced expression of the immunosuppressive molecules CD200 and BTLA as well as IL-10 production by CLL cells.

CONCLUSIONS. Ibrutinib treatment increased the in vivo persistence of activated T cells, decreased the Treg/CD4 ${ }^{+} \mathrm{T}$ cell ratio, and diminished the immune-suppressive properties of CLL cells through BTK-dependent and -independent mechanisms. These features provide a strong rationale for combination immunotherapy approaches with ibrutinib in CLL and other cancers.

TRIAL REGISTRATION. ClinicaITrials.gov NCT01589302 and NCT02029443. Samples described here were collected per OSU-0025.

FUNDING. The National Cancer Institute.

Authorship note: N. Muthusamy and J.C. Byrd contributed equally as co-senior authors to this work

Conflict of interest: K.J. Maddocks received grants and nonfinancial support from Pharmacyclics, Merck, and Bristol-Myers Squibb (BMS), and personal fees from Pharmacyclics, BMS, and Janssen, outside the submitted work. J.A. Jones received personal fees and other support from Janssen, Pharmacyclics, and AbbVie, outside the submitted work. L.A Andritsos received grants from Pharmacyclics during the conduct of the study. F. Awan received grants and personal fees from Pharmacyclics, personal fees from Gilead, and grants from Innate Pharma, outside the submitted work. J.A. Fraietta has a patent for the treatment of cancer using anti-CD19 chimeric antigen receptor licensed to the University of Pennsylvania. C.H. June received grants from Novartis during the conduct of the study and other support from Novartis and Tmunity Therapeutics, outside the submitted work. J.A. Woyach received research funding from Karyopharm Therapeutics, MorphoSys, AbbVie, and Acerta Pharma, outside the submitted work, and is on the advisory board for Janssen. A.J. Johnson has filed a patent on ibrutinib as immune-modulation agent. N. Muthusamy has filed a patent on ibrutinib as an immune-modulation agent. J.C. Byrd has filed a patent on ibrutinib as an immune-modulation agent.

Submitted: August 2, 2016; Accepted: June 1, 2017.

Reference information: / Clin Invest. 2017;127(8):3052-3064.

https://doi.org/10.1172/JCl89756

\section{Introduction}

Chronic lymphocytic leukemia (CLL) patients are characterized by a profound immune suppression and perturbation of both innate as well as adaptive immunity (1), highlighted by increased susceptibility to infections, autoimmune conditions, and a higher incidence of secondary malignancies. In addition to global immunosuppression, CLL cells also evade immune destruction, owing to tumorspecific immune suppression via multiple mechanisms including expression of the immunosuppressive molecules CD200, HLA-G, and programmed death ligand 1 (PD-L1), among others $(2-4)$. Malignant CLL B cells also phenotypically and functionally recapitulate the B10 and B10Pro regulatory B (Breg) cell types that produce IL-10, a major immunosuppressive cytokine (5). In addition to direct tumor immunosuppressive effects, CLL cells can also increase the number of regulatory $\mathrm{T}$ (Treg) cells that diminish cellular immune responses $(6,7)$. Moreover, CLL cells can induce $\mathrm{IDO}^{\text {hi }} \mathrm{CD} 14^{+} \mathrm{HLA}-\mathrm{DR}^{\text {lo }}$ myeloid-derived suppressor cells (MDSCs), which inhibit T cell responses both directly and indirectly 
through promoting Treg cell induction and expansion (8). Collectively, these observations highlight the importance of immune interactions in CLL and make this disease an ideal model to study systemic tumor-mediated immune suppression.

It has been well established that the T cell compartment in CLL patients is dysfunctional. CLL T cell subsets are skewed toward a terminally differentiated phenotype, with significant reduction in naive $\mathrm{T}$ cells and expansion of effector memory/effector $\mathrm{T}$ cells (9). CLL patient $\mathrm{T}$ cells also demonstrate features of pseudoexhaustion, with significant upregulation of checkpoint molecules and exhaustion markers such as programmed death 1 (PD1), CTLA-4, CD160, CD244, and CD57 $(9,10)$. These phenotypic changes are closely associated with profound functional defects including reduced cytotoxic capacity of $\mathrm{CD} 8^{+} \mathrm{T}$ cells and defective immunologic synapse formation (9). A shift in the balance between Th1, Th2, and Th17 immune subsets in CLL patients may also be an important factor in driving disease progression, and the $\mathrm{T}$ cell response in CLL patients is skewed toward Th2 polarization (11). Studies in CLL patients have also shown that a decrease in IL-17producing T cells is associated with Treg cell expansion and disease progression (12-14), while increased Th17 cell numbers correlate with improved overall survival (15). To date, success in therapeutically enhancing cell-mediated immunity in CLL has been limited. Lenalidomide has favorable immune-modulating properties in $\mathrm{T}$ cells in CLL patients via downregulation of the lymphoid transcription factor IKZF1 through a cereblon-dependent mechanism, and can promote durable, sustained complete remissions in $50 \%$ or more of patients receiving this treatment (16). However, lenalidomide in this setting also can produce significant morbidity and sometimes fatal outcome from early-onset tumor flare.

New to the field of B cell cancer therapy are agents that irreversibly target Bruton's tyrosine kinase (BTK) such as ibrutinib, which yields high response rates and durable remissions in patients with CLL. Although data in humans are lacking, multiple reports document the $\mathrm{T}$ cell effects of ibrutinib in mouse models (17-21). Our group recently reported that ibrutinib irreversibly inhibits IL-2inducible T cell kinase (ITK), leading to enhanced Th1 response in vitro and in vivo (21). In a CLL mouse model of bacterial infection, ibrutinib treatment led to a more than 2-fold increase in expansion of Th1 pathogen-specific T cells and corresponding suppression of Th2 immunity (21). Ibrutinib treatment also leads to a shift in macrophages toward a Th1-supportive phenotype and increases CD8 ${ }^{+}$ $\mathrm{T}$ cell tumor infiltration in a mouse model of pancreatic cancer (22).

Recently, our group demonstrated that T cells isolated from CLL patients prior to starting ibrutinib expand ex vivo chimeric antigen receptor (CAR) T cells poorly, whereas those derived during treatment expand significantly better (23). Ruella et al. also reported that addition of ibrutinib to anti-CD19 CAR T cells improves responses against mantle cell lymphoma (24). Furthermore, it has been reported that in mouse lymphoma models ibrutinib enhances $\mathrm{T}$ cell-dependent antitumor immune responses and further potentiates the efficacy of immune checkpoint blockade $(17,18)$. Immune modulatory effects have been preliminarily reported with the BTK inhibitor acalabrutinib (ACP-196), which also demonstrates promising clinical activity in CLL (25). However, unlike ibrutinib, acalabrutinib lacks inhibitory activity against the BTK-related kinase ITK. Clinically, this raises the question of whether more selective BTK inhibition will promote effective immune modulation and avoid the off-target effects observed with ibrutinib.

Here, we comprehensively studied the effects of ibrutinib on $\mathrm{T}$ cells in vivo and the ability of this agent to modulate the immunosuppressive capacity of CLL cells, and compared the results to those achieved with acalabrutinib. Our results indicate that while both agents diminish tumor-mediated immune suppressive molecules, ibrutinib has unique immune-modulating capability in promoting expansion of chronically activated $\mathrm{T}$ cells by diminishing activation-induced cell death.

\section{Results}

Ibrutinib treatment increases numbers of both $C D 4^{+}$and $C D 8^{+} T$ cells via a BTK-independent mechanism. We analyzed serial samples from 19 CLL patients who received ibrutinib treatment on a serial blood procurement study, OSU-0025. Not all experiments were performed on each patient's serial sample; therefore, the actual $n$ for each experiment was less than 19. We first evaluated the absolute numbers of $\mathrm{T}$ cells of various subsets in CLL patients during the course of ibrutinib therapy. As shown in Figure 1A $(n=18)$, a significant increase in total $\mathrm{CD} 4^{+}$and $\mathrm{CD} 8^{+} \mathrm{T}$ cell numbers was observed following ibrutinib treatment (approximately 3-fold by 8 weeks, or the beginning of cycle $3 ; P<0.01$ for both $\mathrm{CD} 4^{+}$ and $\mathrm{CD}^{+} \mathrm{T}$ cells). Both $\mathrm{CD} 4^{+}$and $\mathrm{CD} 8^{+} \mathrm{T}$ cells were further categorized into naive $\left(\mathrm{CD} 45 \mathrm{RA}^{+} \mathrm{CCR} 7^{+}\right)$, central memory (T-CM; $\mathrm{CD}^{2} \mathrm{RA}^{-} \mathrm{CCR}^{+}$), effector memory (T-EM; CD45RA ${ }^{-} \mathrm{CCR} 7^{-}$), and CD45RA ${ }^{+}$effector memory T (T-EMRA) cells (CD45RA ${ }^{+} \mathrm{CCR7}^{-}$) (Figure 1A). The T-EMRA cell subset is considered to consist of more terminally differentiated effector memory/effector $\mathrm{T}$ cells (26). Among the different $\mathrm{T}$ cell subsets, the increase in total cell number was most prominent in the T-EMRA and T-EM compartments, whereas in the naive and $\mathrm{T}-\mathrm{CM}$ subsets, the increase was more modest and not significant at several time points examined. For example, $\mathrm{CD} 4^{+} \mathrm{T}$-EMRA cell numbers increased by more than 3-fold (from $0.055 \times 10^{3}$ to $0.18 \times 10^{3} / \mu \mathrm{l}$ ) 8 weeks into treatment (beginning of cycle 3 ), while naive $\mathrm{T}$ and $\mathrm{CD}^{+} \mathrm{T}-\mathrm{CM}$ cell numbers increased by about 2-fold at the same time point (from 0.065 $\times 10^{3}$ to $0.147 \times 10^{3} / \mu$ l for naive $\mathrm{CD} 4^{+} \mathrm{T}$ cells). This same pattern coincides with the frequencies of different $\mathrm{T}$ cell subsets, where the proportion of both $\mathrm{CD} 4^{+}$and $\mathrm{CD} 8^{+} \mathrm{T}-\mathrm{EM}$ cells was modestly increased by cycle 6 of ibrutinib treatment (Supplemental Figure 1A; supplemental material available online with this article; https://doi.org/10.1172/JCI89756DS1). For example, the mean proportion of $\mathrm{CD}^{+}$T-EMRA cells increased from $5.1 \%$ to $7.3 \%$ $(P<0.05)$. In contrast, the proportion of naive and $\mathrm{T}-\mathrm{CM}$ subsets was modestly but significantly reduced by cycle 6 of treatment in both $\mathrm{CD}^{+}$and $\mathrm{CD}^{+} \mathrm{T}$ cells $\left(P=0.006\right.$ for $\mathrm{CD} 8^{+} \mathrm{T}-\mathrm{CM}$ cells and $P=0.001$ for $\mathrm{CD} 4^{+} \mathrm{T}-\mathrm{CM}$ cells; Supplemental Figure $\left.1 \mathrm{~A}\right)$. The reduced frequencies of both $\mathrm{CD}^{+}$and $\mathrm{CD} 8^{+} \mathrm{T}-\mathrm{CM}$ subsets after ibrutinib treatment appears to be a result of a dilution effect from preferential expansion of the T-EM and T-EMRA subsets as shown in Figure 1A, versus loss of these cells during treatment.

One concern is that the increase in the circulating T cell numbers after ibrutinib treatment may merely reflect the release of $\mathrm{T}$ cells from the secondary lymphoid organs as opposed to true $\mathrm{T}$ cell expansion. To address this, we treated CLL-engrafted mice with ibrutinib and monitored peripheral blood $\mathrm{T}$ cell numbers 
A Patients treated with ibrutinib
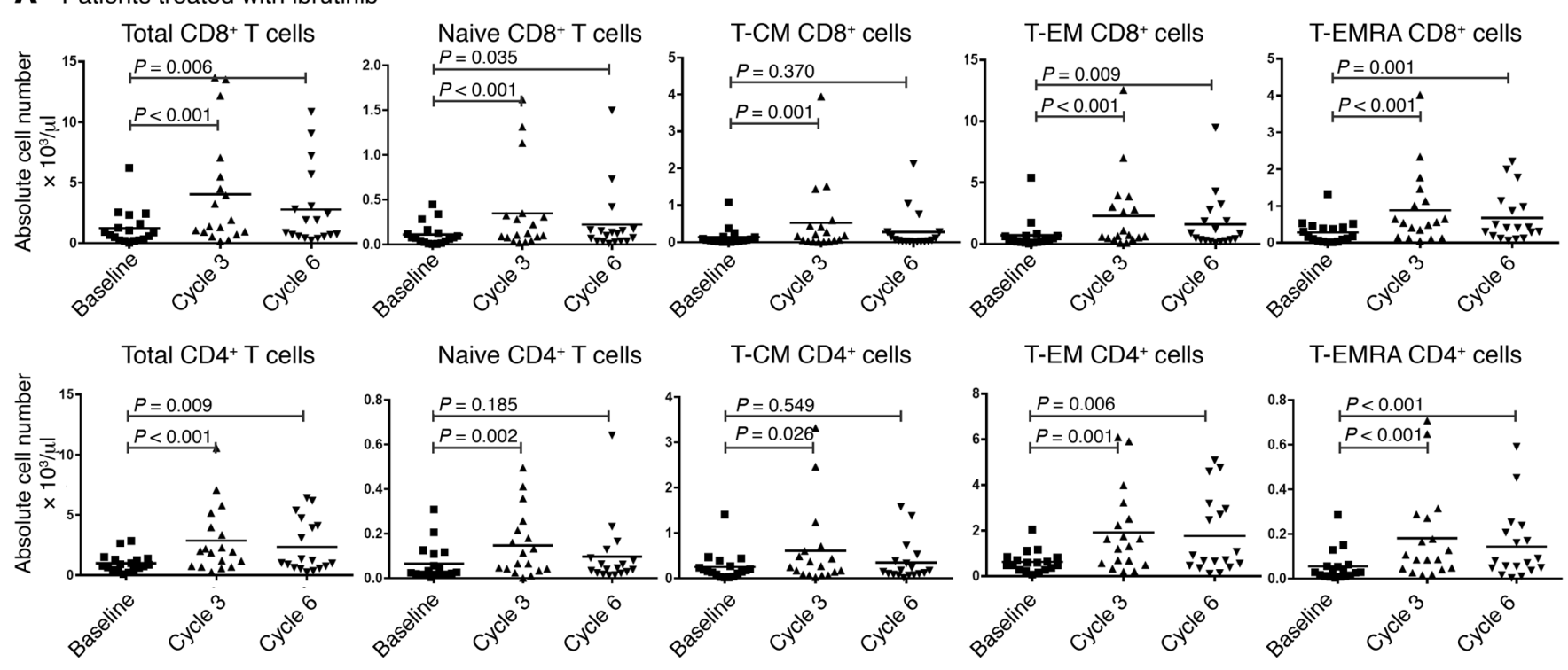

T-EM CD4 ${ }^{+}$cells

T-EMRA CD4 ${ }^{+}$cells

B Patients treated with acalabrutinib

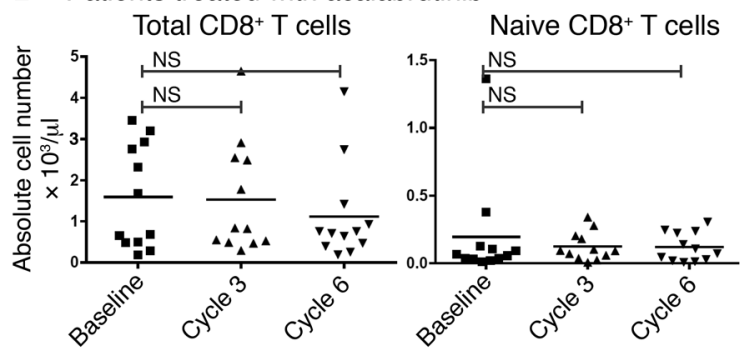

T-CM CD8 ${ }^{+}$cells
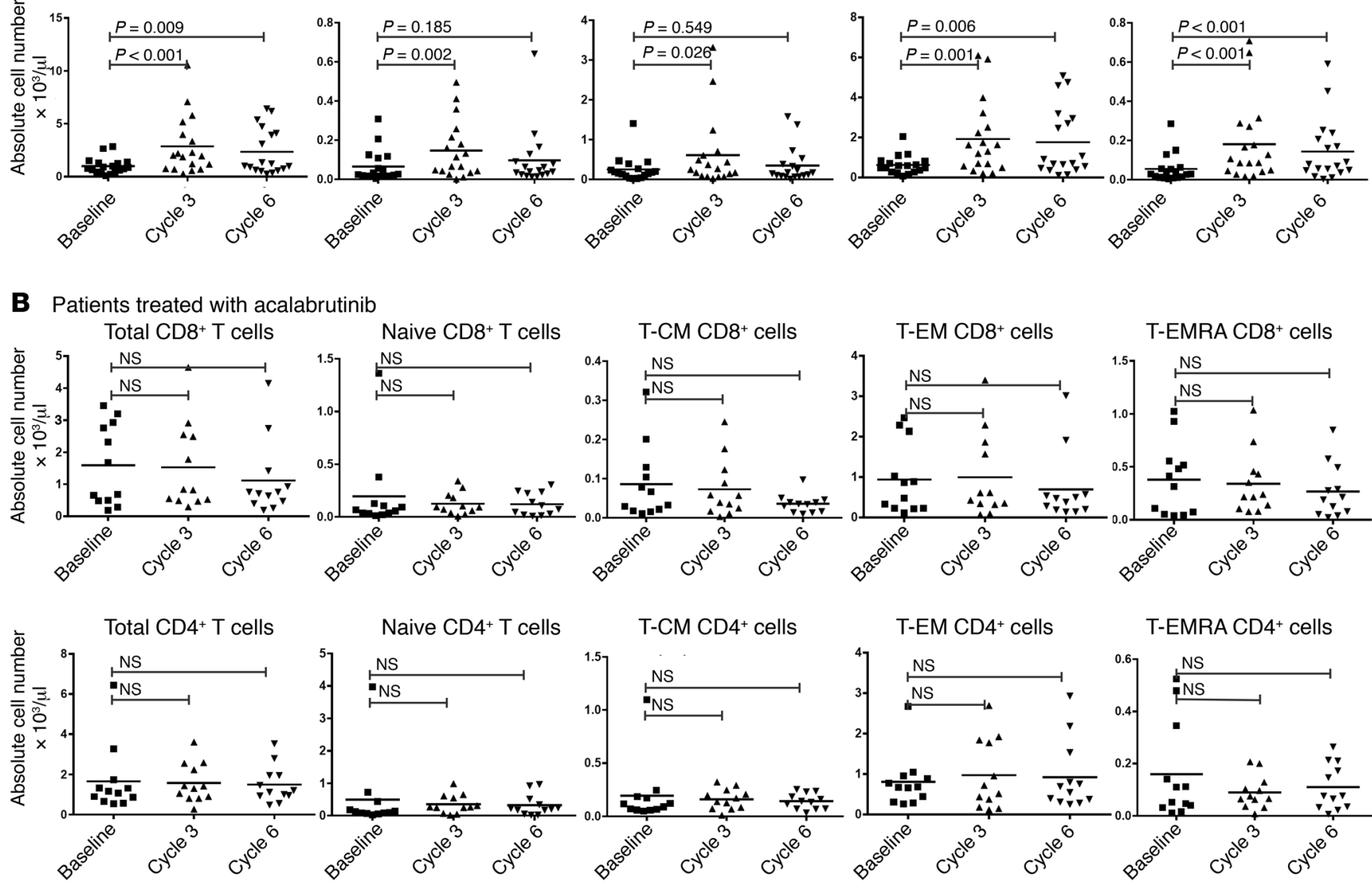

T-EM CD8+ cells

T-EMRA CD8+ cells
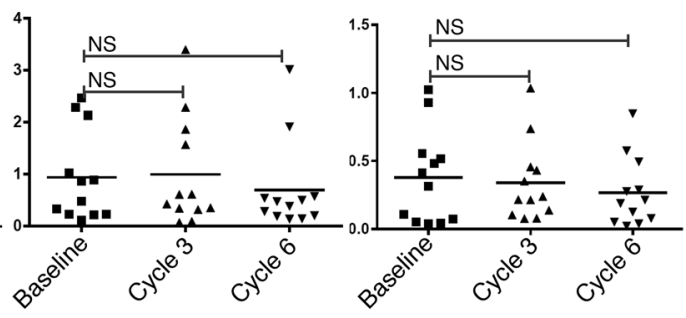

T-CM CD4 ${ }^{+}$cells

T-EM CD4 ${ }^{+}$cells

T-EMRA CD4+ cells
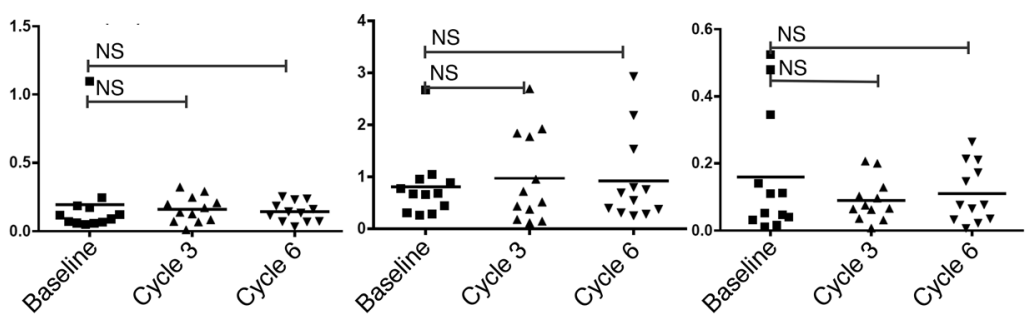

Figure 1. Ibrutinib but not acalabrutinib treatment of CLL patients increases total T cell numbers. (A) Absolute numbers of CD8 $8^{+}$(upper panel) and CD4 ${ }^{+}$ (lower panel) T cells before and during ibrutinib treatment $(n=18)$. (B) Absolute numbers of CD8 ${ }^{+}$(upper panel) and CD4+ (lower panel) T cells before and during acalabrutinib treatment $(n=12)$. Each cycle is 4 weeks. Cycle 3 indicates samples obtained after 2 cycles ( 8 weeks into treatment), and cycle 6 indicates samples obtained after 5 cycles ( 20 weeks into treatment). T cells were differentiated into subsets based on expression of CCR7 and CD45RA: naive T cells (CCR7 ${ }^{+}$CD45RA ${ }^{+}$), central memory T cells (CCR7 ${ }^{+}$CD45RA-), effector memory $T$ cells (CCR7-CD45RA ${ }^{-}$), and more differentiated effector memory T cells (T-EMRA; CCR7-CD45RA ${ }^{+}$). Differences were assessed using linear mixed-effects models. NS, not significant.

before starting ibrutinib, and 2 days and 4 days after starting ibrutinib (Supplemental Figure 7). These time points correspond to the period when CLL cell numbers were transiently increased in peripheral blood after ibrutinib treatment (27). If ibrutinib causes translocation of $\mathrm{T}$ cells from secondary lymphoid organs to peripheral circulation like it does to CLL cells, we would notice an increase in T cell numbers in peripheral blood. However, we found no significant change in circulating $\mathrm{T}$ cell numbers before and after ibrutinib treatment, as shown in Supplemental Figure 7. Furthermore, we went on to question if ibrutinib can enhance the expansion of activated antigen-specific $\mathrm{T}$ cells using a mouse leu- kemia model. As shown in Supplemental Figure 8, we found that ibrutinib increased the number of tumor antigen-specific $\mathrm{T}$ cells in a secondary lymphoid organ (spleen) by approximately 2 -fold.

To further examine if this effect on T cells was dependent or independent of BTK inhibition, we also examined similar parameters in CLL patients treated with acalabrutinib, a more selective BTK inhibitor that does not target other ibrutinib substrates such as ITK (25). Interestingly, we did not find similar increases in the cell numbers after acalabrutinib treatment (Figure 1B, $n=12)$. The frequency of $\mathrm{CD} 4^{+}$and $\mathrm{CD} 8^{+} \mathrm{T}$ cells trended up by cycle 6 , which reflects the decrease in absolute lymphocyte count with 

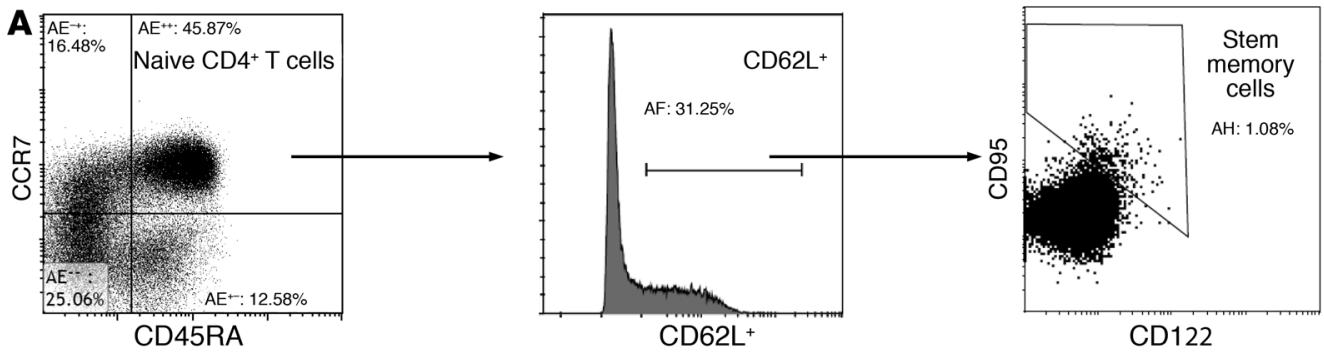

B Healthy donor CLL patients
Gate on $C C R 7^{+}$, CD45RA
Caive $C D 4^{+} T$ cells

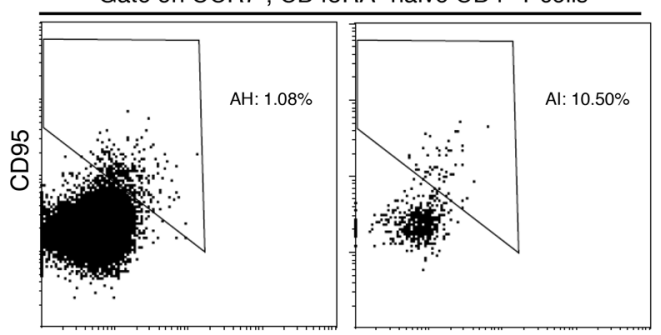

Gate on $\mathrm{CCR}^{+}, \mathrm{CD}^{2} 5 \mathrm{RA}^{+}, \mathrm{CD} 2 \mathrm{~L}^{+}$naive $\mathrm{CD} 4^{+} \mathrm{T}$ cells

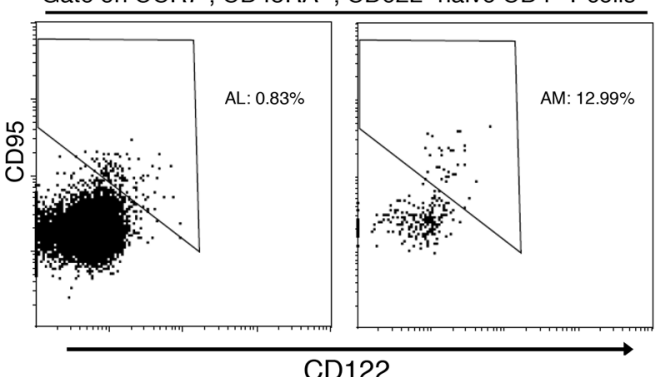

CD122

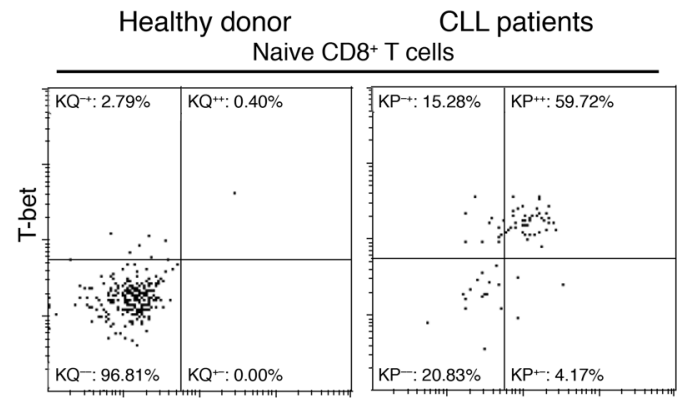

Naive $\mathrm{CD}^{+}{ }^{+} \mathrm{T}$ cells

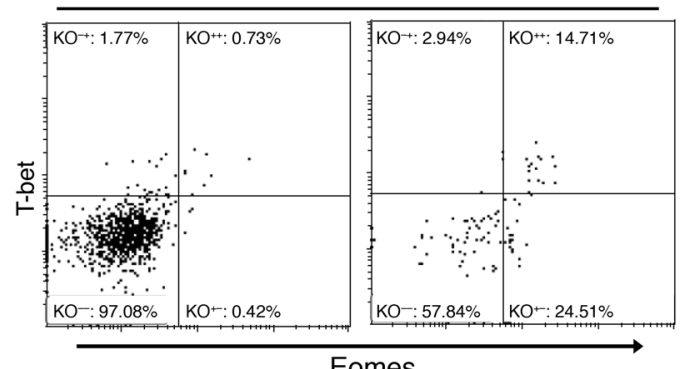

Figure 2. Ibrutinib treatment does not affect total numbers of stem memory $T$ cells. (A) Gating strategy for stem memory T cells. Naive CD4 ${ }^{+} T$ cells (CCR7 ${ }^{+}$CD45RA $\left.{ }^{+}\right)$ expressing CD62L were selected for further analysis. Of these, CD95 + CD122 $2^{\text {hi }}$ cells were defined as stem memory T cells. (B) Left: Representative plots showing stem memory $T$ cells in samples from a healthy donor and a CLL patient. Right: Representative plots showing T-bet and eomesodermin (eomes) expression in naive CD8 ${ }^{+}$and $C D 4^{+} T$ cells from a healthy donor and a CLL patient. (C) Stem memory $T$ cells before and during ibrutinib treatment. Left: Graphs showing absolute numbers (top) and percentages (bottom) of stem memory cells $(n=15)$. Right: Representative plots. Differences were assessed using linear mixed-effects models. NS, not significant.

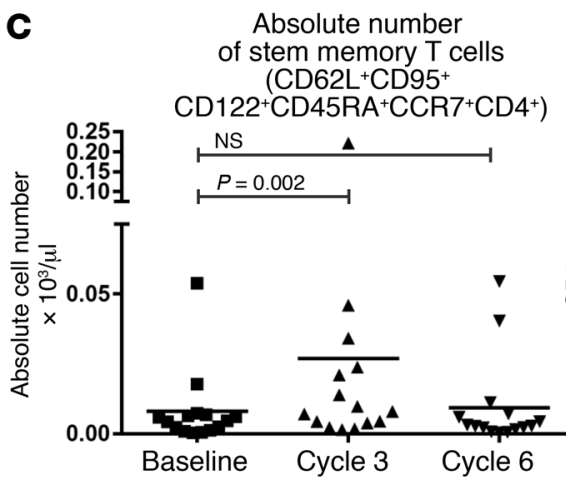

Baseline

Cycle 3

Cycle 6

Gate on $\mathrm{CCR} 7^{+}, \mathrm{CD} 45 \mathrm{RA}^{+}$naive $\mathrm{CD} 4^{+} \mathrm{T}$ cells

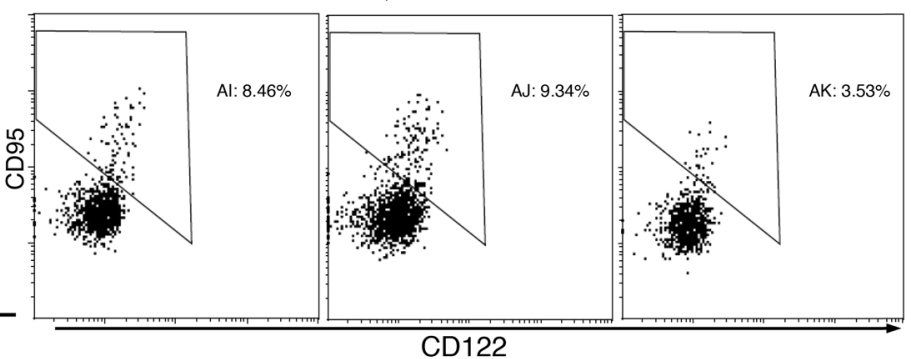

Percentage of stem memory $T$ cells $\left(\mathrm{CD} 95^{+} \mathrm{CD} 122^{+}\right)$among CD62L ${ }^{+}$naive $\mathrm{CD}^{+} \mathrm{T}$ cells

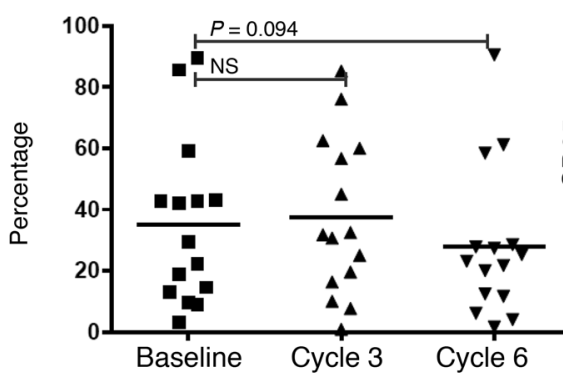

Gate on $\mathrm{CCR}^{+}, \mathrm{CD} 4 \mathrm{RA}{ }^{+}, \mathrm{CD} 62 \mathrm{~L}^{+}$naive $\mathrm{CD} 4^{+} \mathrm{T}$ cells

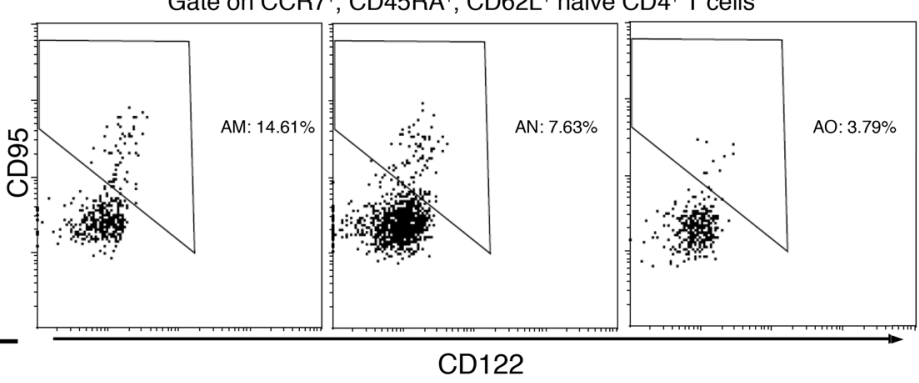


A Patients treated with ibrutinib

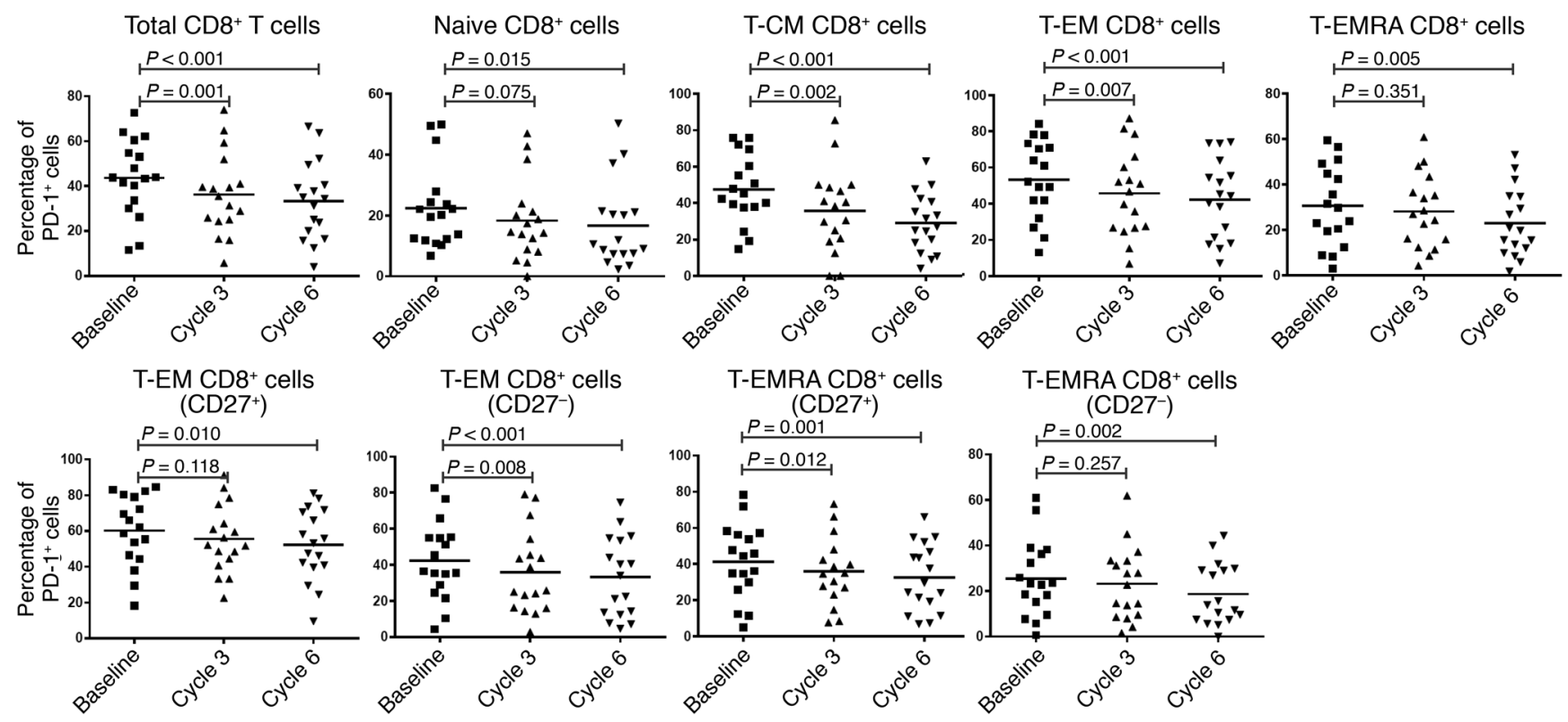

B Patients treated with acalabrutinib

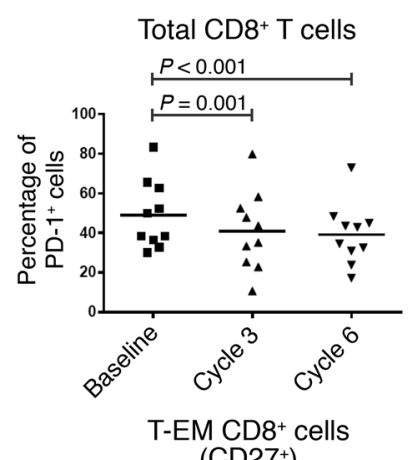

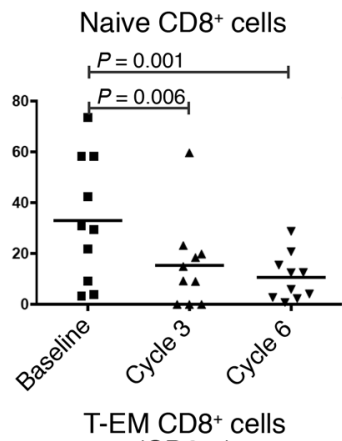

$\left(\mathrm{CD} 27^{-}\right)$

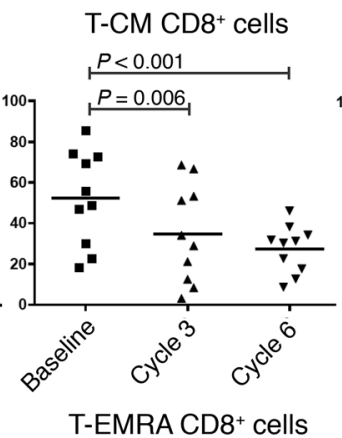

$\left(\mathrm{CD} 27^{+}\right)$

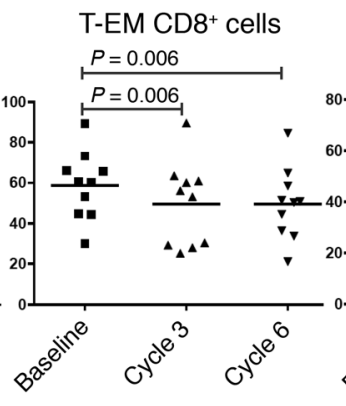

T-EMRA CD8 ${ }^{+}$cells $\left(\mathrm{CD} 27^{-}\right)$
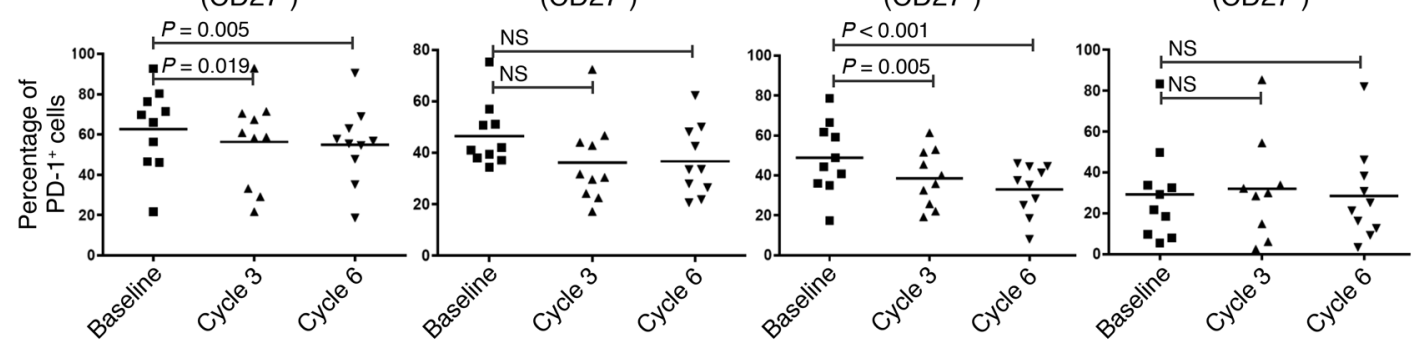

Figure 3. Treatment with BTK inhibitors significantly reduces PD-1-expressing cells in $\mathbf{C D 8}^{+} \mathbf{T}$ cell populations. Percentages of PD-1+ ${ }^{+}$cells among different subsets of CD8 ${ }^{+}$T cells from CLL patients before and during treatment with $(\mathbf{A})$ ibrutinib $(n=17)$ or $(\mathbf{B})$ acalabrutinib $(n=10)$. Differences were assessed using linear mixed-effects models. NS, not significant.

acalabrutinib (Supplemental Figure 1B). Given that activated ITKdeficient $\mathrm{T}$ cells in murine systems have diminished activationinduced cell death (AICD) $(28,29)$, we hypothesized that the ITK inhibitory activity of ibrutinib, but not acalabrutinib, may explain this observation. We therefore examined the influence of these 2 agents on $\mathrm{T}$ cells ex vivo with extended activation. As shown in Supplemental Figure 2, A-C, activated T cells treated with ibrutinib exhibited a dose-dependent decrease in AICD. Upregulation of FAS ligand (FASL) by activated T cells and suicide by FAS-FASL interaction have been found to be an important mechanism for AICD (30). Moreover, ITK inhibition has been reported to impair
AICD by reducing the upregulation of FASL (29). Here, we found that the upregulation of FASL expression in activated T cells after restimulation was indeed decreased by ibrutinib treatment (Supplemental Figure 2C), suggesting that ibrutinib ameliorates AICD by decreasing TCR activation-induced FASL upregulation. In contrast, we did not observe a similar protective effect against AICD with acalabrutinib at these same concentrations.

It has been reported that NK cells can also undergo apoptosis following activation via cytokine receptors (31). Ibrutinib targets such as ITK and BTK also play an important role in NK cell function and signaling processes $(32,33)$. Therefore, we investigated if 
A Patients treated with ibrutinib

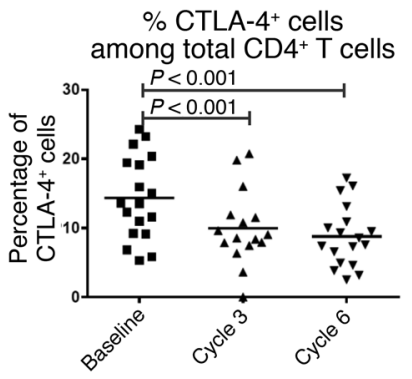

$\%$ CTLA $-4^{+}$cells among $\mathrm{CD}^{2} 5 \mathrm{RA}^{+} \mathrm{CD}^{+} \mathrm{T}^{+}$cells

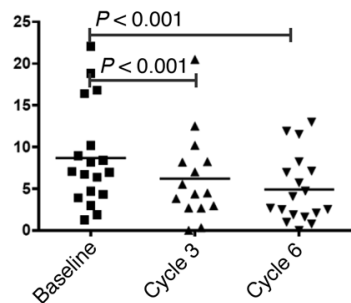

Figure 4. Treatment with BTK inhibitors significantly reduces the frequency of $T$ cells expressing intracellular CTLA-4. Percentages of cells positive for intracellular CTLA-4 expression among total $\mathrm{CD}^{+}{ }^{+} \mathrm{T}$ cells, $\mathrm{CD} 45 \mathrm{RA}^{-} \mathrm{CD}^{+}{ }^{+} \mathrm{T}$ cells, and CD45RA ${ }^{+} \mathrm{CD}^{+}{ }^{+} T$ cells from CLL patients before and during treatment with $(\mathbf{A})$ ibrutinib $(n=18)$ and $(\mathbf{B})$ acalabrutinib $(n=9)$. Differences were assessed using linear mixed-effects models. Data for $\mathrm{CD}^{+} \mathrm{T}$ cells are shown in Supplemental Figure 4.

B Patients treated with acalabrutinib
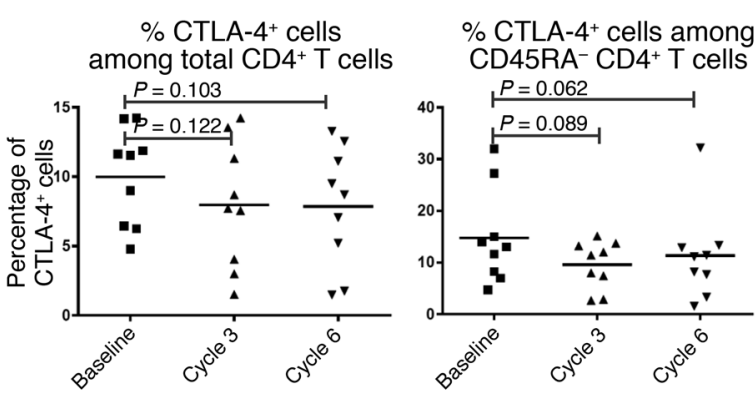

$\%$ CTLA-4 $4^{+}$cells among CD45RA ${ }^{+}$CD4 ${ }^{+} \mathrm{T}$ cells

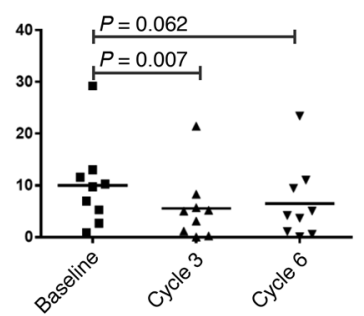

ibrutinib can also ameliorate AICD of NK cells utilizing an established assay system in which NK cells are costimulated with IL-2/ IL-12 or IL-15/IL-12. Here, we found a similar protection by ibrutinib, but not acalabrutinib (Supplemental Figures 2 and 9).

Ibrutinib treatment does not compromise stem memory $T$ cells localized in the naive $T$ cell compartment. We next analyzed stem memory $\mathrm{T}$ (TSCM) cells, the long-lasting memory $\mathrm{T}$ cells that share many surface marker profiles with naive T cells. We further differentiated TSCM from naive T cells by staining for CD122 and CD95, as shown in Figure 2A. Interestingly, we found that CLL patients have more (>10\%) TSCM cells within the naive T cell gate (Figure 2B). This differs from healthy donors, who usually have less than approximately $1 \%-2 \%$ of TSCM cells among the naive T cell gate (34). T-bet and eomesodermin are established Th1 differentiation markers (35). As expected, we found that few naive T cells from healthy donors express these markers (Figure 2B). However, a significant percentage of naive $\mathrm{T}$ cells (more than $10 \%$ naive CD $4^{+}$cells) from CLL patients express T-bet or eomesodermin. Therefore, a significant percentage of naive $\left(\mathrm{CCR} 7^{+} \mathrm{CD} 45 \mathrm{RA}^{+}\right)$ $\mathrm{T}$ cells from CLL patients are not bona fide naive $\mathrm{T}$ cells, but rather TSCM cells. We found that in patients receiving ibrutinib, the absolute number of TSCM cells was increased by cycle 3 and was not significantly changed by cycle 6 (Figure 2C, $n=15$ ).

Frequencies of PD-1- and CTLA-4-positive $T$ cells are significantly reduced after ibrutinib treatment. A key feature of T cells from CLL patients is the high percentage of cells expressing checkpoint inhibitor molecules such as PD-1 (Supplemental Figure 6). As ibrutinib rescues AICD of T cells, it is possible that these rescued cells may express a higher level of exhaustion markers that would suggest less immunologic potential. We therefore examined these markers in different $\mathrm{T}$ cell subsets before and after ibrutinib treatment. We found no significant changes in CD244, CD160, or CD57 after ibrutinib treatment (data not shown). However, the percentages of PD-1- positive cells among total $\mathrm{CD}^{+}$(Figure $3 \mathrm{~A}, n=17$ ) and total CD4 ${ }^{+}$ (Supplemental Figure 3A, $n=17$ ) T cells were significantly reduced. The decrease in PD- 1 was statistically significant by cycle 3 for $\mathrm{CD}^{+}$ T cells $(P=0.001)$, and by cycle 6 for both $\mathrm{CD}^{+}(P=0.001)$ and $\mathrm{CD}^{+}(P<0.001) \mathrm{T}$ cells. We also noticed that although PD-1 positivity was reduced in all the $\mathrm{T}$ cell subsets among both $\mathrm{CD} 4^{+}$and $\mathrm{CD} 8^{+}$ $\mathrm{T}$ cells, the reduction was most dramatic in the T-CM compartment (Figure 3A and Supplemental Figure 3A). CD27 was downregulated in the more differentiated effector $\mathrm{T}$ and T-EM cells, prompting us to further differentiate the T-EM and T-EMRA cells into $\mathrm{CD} 27^{+}$versus CD27- populations. We did not find significant differences in the distributions of $\mathrm{CD} 27^{+}$and $\mathrm{CD} 27^{-} \mathrm{T}$ cell subsets. However, for $\mathrm{CD} 4^{+}$ $\mathrm{T}$ cells, the $\mathrm{CD} 27^{+} \mathrm{T}-\mathrm{EM}$ and T-EMRA cells showed a statistically significant reduction in PD-1 after ibrutinib treatment $(P<0.001)$, while their $\mathrm{CD}^{2} 7^{-}$counterparts did not (Supplemental Figure 3).

In our serial samples we were able to detect CTLA- 4 expression by intracellular staining (Figure $4 \mathrm{~A}$ and Supplemental Figure 4A, $n=18$ ). We again detected a significant reduction in intracellular CTLA $-4^{+}$cells among both CD $4^{+} \mathrm{T}$ cells (Figure $4 \mathrm{~A} ; \mathrm{P}<0.001$ for both cycle 3 and cycle 6 ) and $\mathrm{CD}^{+} \mathrm{T}$ cells (Supplemental Figure 4A; $P<0.01$ for both cycle 3 and cycle 6), with the reduction being more dramatic in $\mathrm{CD}^{+} \mathrm{T}$ cells. Cells from patients treated with acalabrutinib showed similar patterns of change in PD-1 expression (Figure $3 \mathrm{~B}$ and Supplemental Figure 3B, $n=10$ ). Acalabrutinib treatment also reduced CTLA- 4 expression on T cells (Figure 4B and Supplemental Figure 4B, $n=9$ ), suggesting that ibrutinib affects PD- 1 and CTLA-4 expression in T cells indirectly by inhibiting BTK.

Ibrutinib treatment does not significantly change $T$ cell polarization. We previously reported that ibrutinib enhances Th1 polarization in vitro and in vivo in murine models. To study $\mathrm{T}$ cell polarization in human CLL patients, we restimulated peripheral blood mononuclear cells (PBMCs) from ibrutinib-treated patients with PMA/ionomycin and assessed their cytokine production pro- 


\section{A Patients treated with ibrutinib}

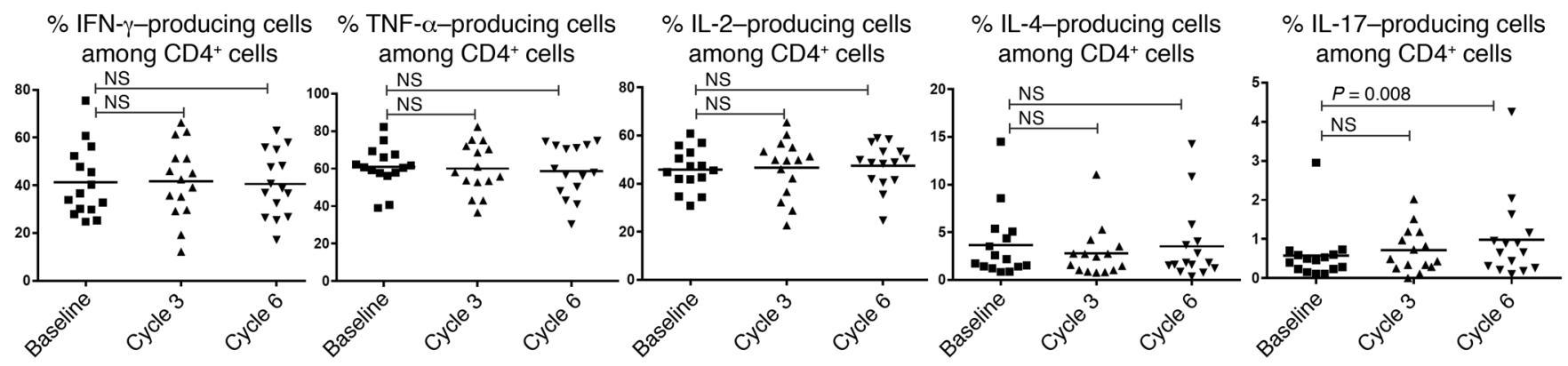

B Patients treated with acalabrutinib

$$
\begin{array}{ccc}
\% \text { IFN- } \gamma \text {-producing cells } & \% \text { TNF- } \alpha \text {-producing cells } & \% \text { IL-2-producing cells } \\
\text { among CD } 4^{+} \text {cells } & \text { among CD4 } 4^{+} \text {cells } & \text { among CD } 4^{+} \text {cells }
\end{array}
$$

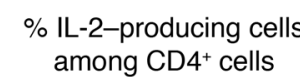
$\%$ IL-4-producing cells
among CD4 ${ }^{+}$cells
$\%$ IL-17-producing cells among $\mathrm{CD}^{+}$cells
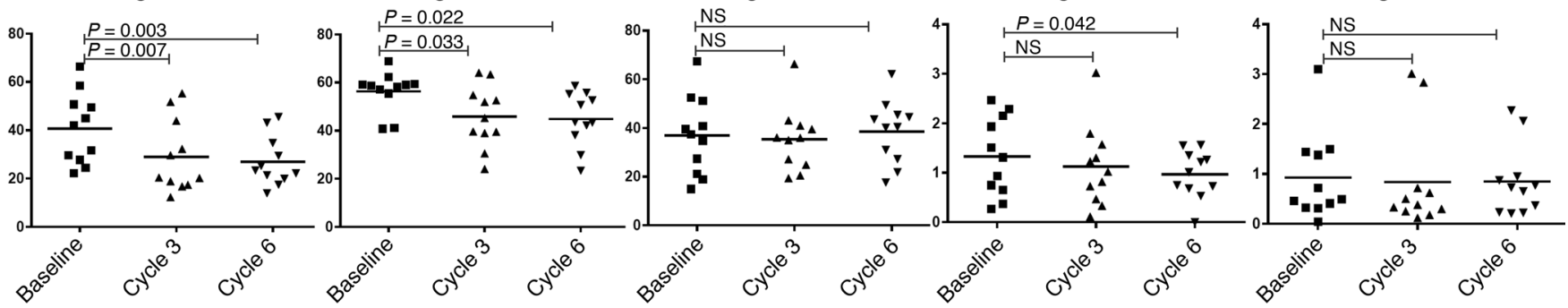

Figure 5. Ibrutinib treatment does not affect T cell production of Th1 or Th2 cytokines but moderately increases frequency of Th17 cells. PBMCs from CLL patients before and during treatment with $(\mathbf{A})$ ibrutinib $(n=15)$ or $(\mathbf{B})$ acalabrutinib $(n=11)$ were stimulated with PMA/ionomycin for 5 hours. Production of IFN- $\gamma$, TNF, IL-2, IL-4, and IL-17 was detected by intracellular cytokine staining. Percentages of cytokine-producing cells are shown. Differences were assessed using linear mixed-effects models. NS, not significant.

file (representative dot plots shown in Supplemental Figure 5). As shown in Figure 5A $(n=15)$, ibrutinib treatment did not alter Th1 (IFN- $\gamma$ ), Th2 (IL-4), and Th0 (IL-2 and TNF) cytokine expression profiles. However, there was a noticeable increase in the percentage of cells producing IL-17 in patients who received ibrutinib treatment $(P=0.008$, Figure $5 \mathrm{~A})$. In contrast, acalabrutinib-treated patients did not have a posttreatment change in the frequency of IL-17producing cells (Figure 5B, $n=11$ ). These results indicate that inhibition of non-BTK targets such as ITK play a key role in the expansion of Th17 cells. The average percentage of IFN- $\gamma^{+}, \mathrm{IL}^{-} 4^{+}$, and $\mathrm{TNF}^{+}$ cells showed a decreasing trend after treatment with acalabrutinib (Figure 5B) compared with ibrutinib (Figure 5A), indicating a BTKbut not ITK-dependent mechanism.

Ibrutinib decreases the Treg/CD4 ${ }^{+} T$ cell ratio but not absolute number of $\mathrm{CD} 25^{+} \mathrm{Foxp}^{+}$Treg cells. In vitro experiments have shown enhanced differentiation toward Foxp $3^{+}$Treg cells by ITK-deficient conventional $\mathrm{CD}^{+} \mathrm{T}$ cells (36). Therefore, we sought to determine how ibrutinib treatment in human CLL patients affects Treg cells in vivo. Treg cells were identified as $\mathrm{CD} 4^{+} \mathrm{CD} 25^{+} \mathrm{Foxp}^{+}$cells (Figure $6 \mathrm{~A})$. We found an approximately 2 -fold reduction in the percentage of $\mathrm{CD}^{2} 5^{+} \mathrm{Foxp}^{+}$Treg cells among $\mathrm{CD} 4^{+} \mathrm{T}$ cells by cycle $3(P<0.001)$ that persisted to cycle $6(P<0.001)$ of ibrutinib treatment (Figure 6B, $n=18)$. However, this reduction in the Treg/CD $4^{+} \mathrm{T}$ cell ratio was associated with an increase in the absolute number of $\mathrm{CD}^{+} \mathrm{T}$ cells and the total number of Treg cells remained unchanged (Figure 6B). In acalabrutinib-treated patients, the number of $\mathrm{CD} 4^{+} \mathrm{CD} 25^{+} \mathrm{Foxp} 3^{+}$ Treg cells and the Treg/CD4 ${ }^{+} \mathrm{T}$ cell ratio was not significantly changed (Figure 6C, $n=11$ ).
Ibrutinib downregulates immunosuppressive molecules CD2OO and BTLA on CLL cells. CLL cells have been reported to express a variety of immunosuppressive ligands; we therefore evaluated the expression levels of these immunosuppressive molecules in CLL cells before and after ibrutinib treatment. The surface expression of PD-L1, HLA-G, and CD276 was low in general, and we did not detect a significant change in their expression in CLL cells after ibrutinib treatment (data not shown). However, CD200 expression was significantly reduced as early as cycle 3 of ibrutinib treatment, and this reduction persisted through cycle $6(P<0.001$ for both; Figure 7A, $n=18$ ). BTLA expression was not significantly changed in $\mathrm{T}$ cell subsets (data not shown), while significant reduction in CLL cells was observed ( $P<0.001$ for both, $n=16)$. Similar reductions were observed in samples from patients treated with acalabrutinib (Figure 7B, $n=12$ ), indicating a BTK-dependent mechanism for these changes.

The capacity of CLL cells to make IL-10 after prolonged B1OPro conditioning is impaired after ibrutinib treatment. It has been reported that CLL cells share phenotypic and functional features with Breg cells and can produce IL-10 after in vitro stimulation under B10 (5-hour stimulation) or B10Pro (48-hour stimulation) conditions (5). Activation through the BCR, TLRs, and CD40 is required for production of IL-10 by B cells or CLL cells (37). As BTK is involved in signal transduction of all these receptors $(38,39)$, we hypothesized that BTK inhibition may affect IL-10 production in CLL cells. We detected significant IL-10 production by CLL cells after a brief in vitro stimulation under B10 conditions in less than half of the CLL patient 


\section{Table 1. Clinical characteristics of the studied patients}

\begin{tabular}{|c|c|}
\hline Characteristics & Value \\
\hline \multicolumn{2}{|l|}{ Age at diagnosis } \\
\hline Mean (SD) & $54(11.5)$ \\
\hline Median & 53 \\
\hline \multicolumn{2}{|l|}{ Age at starting ibrutinib } \\
\hline Mean (SD) & $64(8.6)$ \\
\hline Median & 64 \\
\hline Male sex & 11 \\
\hline \multicolumn{2}{|l|}{ Rai stage at ibrutinib treatment } \\
\hline $0-2$ & 1 \\
\hline 3 & 1 \\
\hline 4 & 17 \\
\hline \multicolumn{2}{|l|}{ Number of prior therapies } \\
\hline 0 & 0 \\
\hline 1 & 6 \\
\hline 2 & 4 \\
\hline $3-4$ & 4 \\
\hline 5 or more & 5 \\
\hline Prior allogeneic transplant & 2 \\
\hline \multicolumn{2}{|l|}{ Cytogenetics } \\
\hline $\operatorname{Del}(13 q)$ & 13 \\
\hline $\operatorname{Del}(11 q)$ & 9 \\
\hline $\operatorname{Del}(17 q)$ & 4 \\
\hline Trisomy 12 & 0 \\
\hline Complex karyotype & 8 \\
\hline $\begin{array}{l}\text { IgVH mutation status, unmutated, } \\
\text { mutated, unknown }\end{array}$ & $14,5,0$ \\
\hline $\begin{array}{l}\text { Bone marrow involvement at the time } \\
\text { of ibrutinib therapy: \% (range) }\end{array}$ & $88.1(70-99)$ \\
\hline \multicolumn{2}{|l|}{ ALC (baseline before ibrutinib) } \\
\hline Mean (SD) & $46.5(40.1)$ \\
\hline Median & 29.9 \\
\hline \multicolumn{2}{|l|}{ ALC (cycle 3 day 1 ) } \\
\hline Mean (SD) & $122.6(66.9)$ \\
\hline Median & 99.1 \\
\hline \multicolumn{2}{|l|}{ ALC (cycle 6 day 1 ) } \\
\hline Mean (SD) & $60.9(52.9)$ \\
\hline Median & 47.6 \\
\hline
\end{tabular}

samples, in agreement with previous reports (5), and we did not detect significant changes in the frequency of these rare malignant B10 cells after ibrutinib treatment (Figure 8, A and B, $n=13$ ). However, by stimulating cells under B10Pro conditions, we were able to induce IL-10 production by CLL cells from most of the samples tested. Interestingly, after ibrutinib treatment, CLL cells showed a dramatically reduced capacity to secrete IL-10 after being stimulated under B10Pro conditions $(P<0.001)$. This reduction was observed as early as cycle 3 of ibrutinib treatment, when the majority of the patients studied still showed transient lymphocytosis (Figure $8 \mathrm{~B}, n=18$ ). Samples from patients treated with acalabrutinib showed similar results (Figure $8 \mathrm{C}, n=10$ for B10 conditions, $n=12$ for B10Pro condition), indicating that the reduced capacity of CLL cells to make IL-10 following ibrutinib treatment is from BTK inhibition.

\section{Discussion}

Numerous studies in mice have reported the favorable immunemodulating effect of ibrutinib that likely occurs through multiple mechanisms (17-21). Here, in what we believe is the first comprehensive human study of ibrutinib's effects on T cells, we identified significant increases in both $\mathrm{CD}^{+}$and $\mathrm{CD}^{+} \mathrm{T}$ cell numbers following ibrutinib treatment in CLL patients. These $\mathrm{T}$ cells, while increased in number following ibrutinib treatment, lack the typical immunophenotypic features of CLLexhausted $\mathrm{T}$ cells, as evidenced by significantly lower PD-1 and intracellular CTLA-4 expression. Unlike other immunemodulating agents such as IL-2 that expand Treg cells, ibrutinib in fact decreases the Treg/CD $4^{+} \mathrm{T}$ cell ratio by selectively expanding conventional $\mathrm{T}$ cells. Separate from the favorable effects on $\mathrm{T}$ cells, ibrutinib also modulates the expression of several immune-suppressive molecules on/in CLL cells including CD200, BTLA4, and IL-10. Acalabrutinib is a secondgeneration, selective BTK inhibitor. As shown in Supplemental Table 1, while ibrutinib has comparable IC50 for BTK and ITK, acalabrutinib has virtually no affinity for ITK $(25,40)$. Our pharmacologic studies in patients clearly differentiate ibrutinib from the more selective BTK inhibitor acalabrutinib in its ability to inhibit AICD via ITK inhibition. Collectively, this and previous studies show that ibrutinib represents a potentially novel T cell immune-modulating agent, and our data clearly differentiate it from other immunotherapeutics used in cancer.

The remarkable finding of our work is the notable increase of both $\mathrm{CD}^{+}$and $\mathrm{CD} 8^{+} \mathrm{T}$ cells in patients receiving ibrutinib. This $\mathrm{T}$ cell expansion is unlikely to be caused by BTK inhibition, as we did not observe increased $\mathrm{T}$ cell numbers in patients treated with the more selective BTK inhibitor acalabrutinib that lacks ITK inhibitory activity. While discerning the mechanism of T cell expansion in vivo in CLL patients is not possible, we provide evidence that ibrutinib treatment of activated T cells diminishes AICD by targeting ITK, a finding also reported in murine models of ITK deficiency (29). ITK has been demonstrated to be involved in TCR-induced upregulation of FASL and AICD of T cells $(28,29)$. In vitro, ITK-deficient T cells have been found to have impaired proliferation (41), whereas in vivo, activated $I T K^{-/} \mathrm{T}$ cells survived to a much greater degree than normal T cells, leading to a greater accumulation (28). Targeting ITK with kinase inhibitors showed a similar pattern. In vitro, ITK inhibitors inhibit IL-2 secretion and T cell proliferation (42), whereas in vivo the ITK inhibitor was found to reduce AICD, leading to a 2- to 3-fold increase in activated T cell numbers (29).

In contrast to our findings, Niemann et al. (43) recently reported that ibrutinib treatment led to a decrease in circulating T cell numbers in CLL patients that paralleled the progressive decrease of CLL tumor burden and normalization of $\mathrm{T}$ cell counts in most patients. This difference might be best explained by the impact of CLL tumor burden. It has been postulated that CLL cells promote chronic stimulation of $\mathrm{T}$ cells and lead to an exhaustion phenotype by inducing a CLL-specific immune response, or by modifying the $\mathrm{T}$ cell response to chronic infections including cytomegalovirus (CMV) $(10,44)$. In either case, CLL cells cause chronic activation of T cells, and it is the activated $\mathrm{T}$ cells, but not resting $\mathrm{T}$ cells, that are susceptible to AICD and can be rescued by ibrutinib. Therefore, this effect on $\mathrm{T}$ cell numbers by ibrutinib is likely only to be seen in patients who 


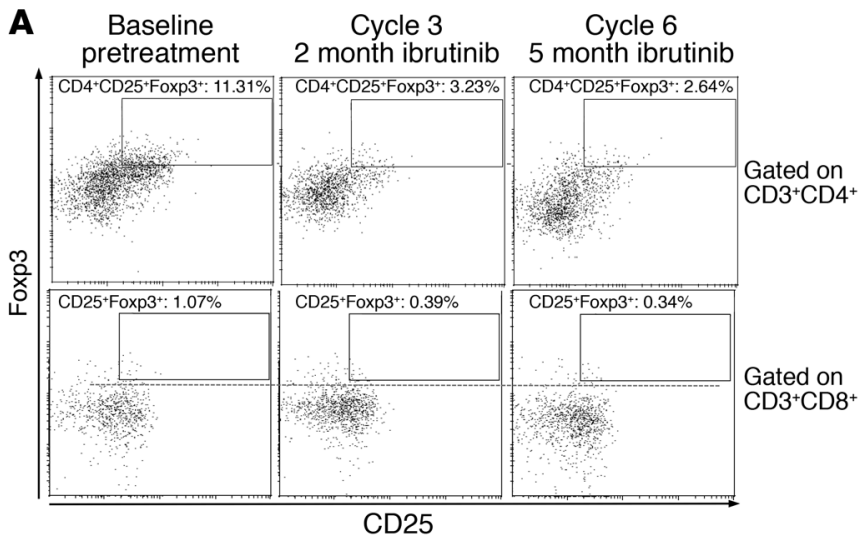

B Percentage of CD25 ${ }^{+}$Foxp3 $^{+}$cells (gated on $\mathrm{CD}^{+} \mathrm{CD} 4^{+} \mathrm{T}$ cells)
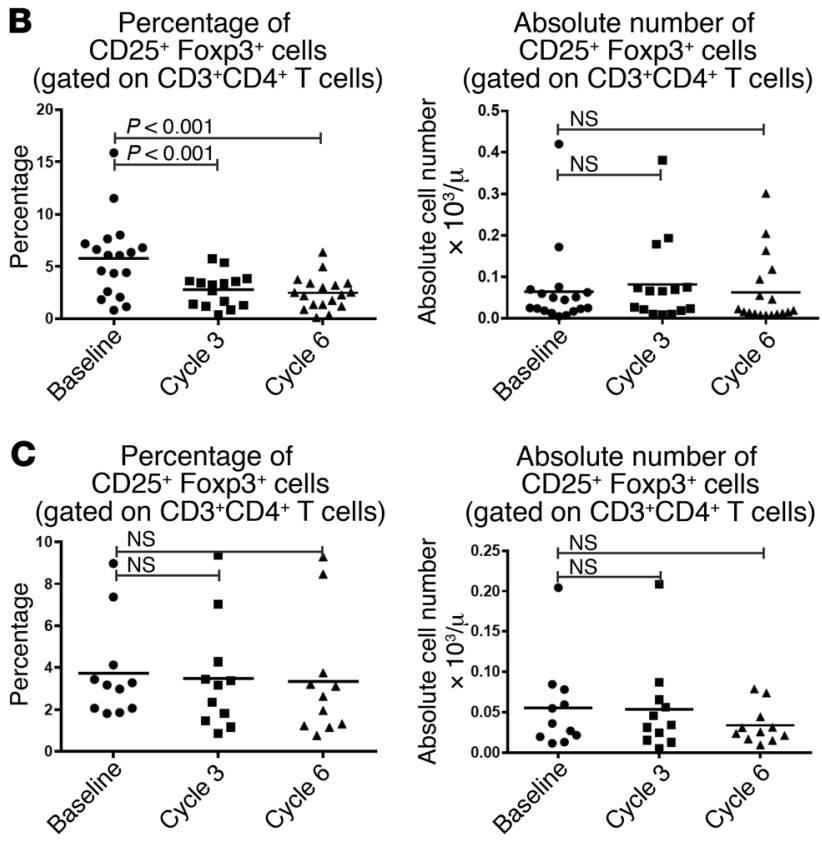

still have significant tumor burden, since those patients who have achieved remission will no longer have CLL-induced aberrant activation of $\mathrm{T}$ cells. To more selectively study the direct impact of BTK inhibition on T cells, we selected earlier time points (8 and 20 weeks into treatment) and studied patients with persistent lymphocytosis (absolute lymphocyte counts at cycle 1 and cycle 6 are comparable; Table 1) to address this confounding factor. Consistent with this hypothesis, in mouse models, our group reported that ibrutinib strongly increased the number of activated $\mathrm{T}$ cells during listeria infection (21), while it has no significant impact on the resting T cell populations from healthy noninfected mice. Furthermore, in ibrutinib-treated patients, we found that the increase in $\mathrm{T}$ cell numbers was most prominent in the effector $\mathrm{T}$ and T-EM cells compared with the resting naive $\mathrm{T}$ and $\mathrm{T}-\mathrm{CM}$ cells. As a side note, it is possible that some of the changes in $\mathrm{T}$ cell populations are due to redistribution rather than expansion, as has been observed in HIV patients after institution of antiretroviral therapy (45). However, the durable effects we observed make it unlikely that all the effects that we describe here are due to redistribution. Moreover, our animal experiments (Supplemental Figures 7 and 8, and ref. 21) provided evidence that ibrutinib treatment did lead to a bona fide increase in
Figure 6. Ibrutinib treatment of CLL patients leads to a reduced frequency, but not reduced absolute number, of $\mathrm{CD} 4^{+} \mathrm{CD} 25^{+}$Foxp $3^{+}$Treg cells. (A) Representative plots showing CD25 and intranuclear Foxp3 staining in $\mathrm{CD}^{+} \mathrm{CD}^{+}$(upper panel) and $\mathrm{CD}^{+} \mathrm{CD3}^{+}$(lower panel) T cells. (B and C) Percentages (left) and absolute numbers (right) of $\mathrm{CD} 25^{+} \mathrm{Foxp} 3^{+}$regulatory T cells among total $C D 4^{+} T$ cells before and during treatment with (B) ibrutinib $(n=18)$ or $(\mathbf{C})$ acalabrutinib $(n=11)$. Differences were assessed using linear mixed-effects models. NS, not significant.

the numbers of activated antigen-specific T cells in secondary lymphoid organs, while it did not cause significant translocation of $\mathrm{T}$ cells from secondary lymphoid organs to the peripheral circulation.

We found that ibrutinib treatment leads to preferential expansion of more differentiated T cell subsets (e.g., T-EM and EMRA), but it does not have a deleterious effect on the absolute number of naive $\mathrm{T}$ and $\mathrm{T}-\mathrm{CM}$ cells. This is another feature that is desired for cancer immunotherapy. In contrast, although IL-2 is able to increase effector cell proliferation it also compromises the persistence of the less differentiated memory $\mathrm{T}$ cells, and therefore has a deleterious effect on the long-term persistence of antitumor immunity (46). We also demonstrated that ibrutinib treatment does not compromise the total numbers of the TSCM cells, which represent the earliest and long-lasting memory $\mathrm{T}$ cells. The selfrenewal capacity and long-term survival of these cells make them an ideal vehicle for cancer immunotherapy. Furthermore, while $\mathrm{T}$ cells from CLL patients demonstrate features of exhaustion similar to those exposed to chronic stimulation by viral infections (44) and ibrutinib preferentially increases the number of these exhausted T cells from AICD, we did not see enrichment of such cells. Instead, we detected diminished PD-1 surface and intracellular CTLA-4 expression.

Previous work from our group and others demonstrated in vivo Th1 skewing $(21,22)$ with ibrutinib treatment in murine models. Here, we observed no significant changes in the percentage of T cells expressing intracellular Th1 (IFN- $\gamma$ ) or Th2 (IL-4) cytokines in CLL patients treated with ibrutinib. However, we found a moderate increase in the frequency of $\mathrm{T}$ cells capable of producing IL-17 (Th17 cells). Ibrutinib has also been recently found to enhance IL-17 responses indirectly by modulating the function of antigen-presenting cells such as dendritic cells (47). In vivo, Th17 cells have been found to undergo FAS-mediated AICD (48), a process that could also be blocked by ITK inhibition. Our findings suggest that the net effect of ibrutinib treatment in CLL patients is the increased percentage of Th17 cells. There is accumulating evidence that a Th17 response may play a role in CLL pathogenesis. Decreased frequency of Th17 cells has been found to be associated with Treg cell expansion and disease progression in CLL patients (12-14). In contrast, elevated Th17 cells in CLL patients is associated with improved survival (15).

The influence of ibrutinib in CLL patients also has direct positive influence on the immunosuppressive capacity of the primary tumor cells. In this study, we confirmed that CD200 and BTLA are significantly downregulated on the surface of CLL cells as early as cycle 3 of ibrutinib treatment. While the function of BTLA on CLL cells is uncertain, CD200 regulates both innate and adaptive immunity and plays a key role in both tumor-specific and global immune suppression in CLL patients (4). Moreover, CD200 expression on tumor cells has been found 
CD200 expression level

A Ibrutinib treatment

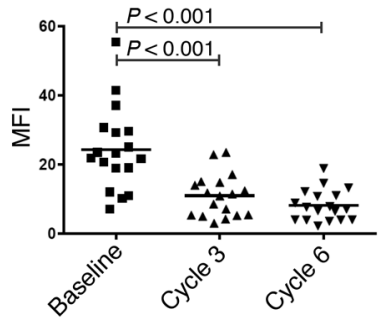

B Acalabrutinib treatment

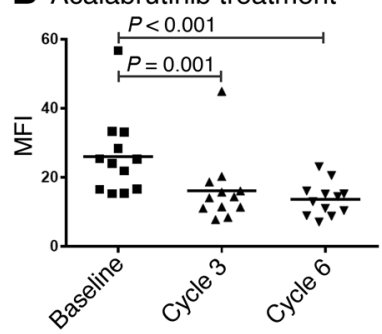

BTLA expression level
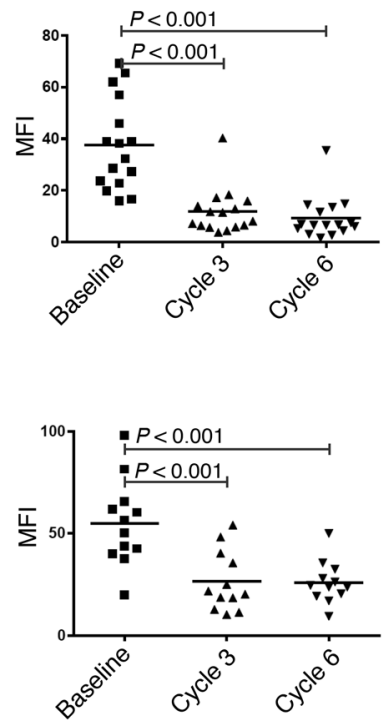

Figure 7. Ibrutinib and acalabrutinib treatment of CLL patients reduces CD200 and BTLA expression in CLL cells. Left panels: CD200 expression level as measured in mean fluorescence intensity (MFI) on CLL cells $\left(\mathrm{CD}_{19}{ }^{+} \mathrm{CD5}^{+}\right)$before and during treatment with $(\mathbf{A})$ ibrutinib $(n=18)$ or $(\mathbf{B})$ acalabrutinib $(n=12)$. Right panels: BTLA expression level as measured in MFI on CLL cells before and during treatment with $(\mathbf{A})$ ibrutinib $(n=16)$ or (B) acalabrutinib $(n=12)$. Differences were assessed using linear mixedeffects models.

to promote the expansion of Treg cells, and CD200 blockade significantly decreases Treg cell numbers $(6,7,49)$. Ibrutinib treatment of CLL patients dramatically reduced the frequency of malignant B10Pro cells, which can express IL-10 after prolonged in vitro stimulation, and similar findings with acalabrutinib suggest this is a BTK-dependent effect. To induce IL-10 expression in B10/B10Pro cells, stimulation via BCR, TLR4/9, and CD4OL are required (5). BTK is involved in signaling transduction of all these receptors $(39,50,51)$. Recently, it was found that the chemokine CXCL12 enhances IL-10 production in CLL cells via the CXCR4/STAT3 pathway (38), and BTK inhibition was reported to impair CXCR4 surface expression and signaling in CLL cells (52). In support of this, mice with BTK deficiency (XID mice) showed a more severe reduction in the numbers of B1a cells (53), which are also $\mathrm{CD}^{+} \mathrm{B}$ cells and are enriched with B10 ( $\left.30 \%\right)$ and B10Pro (30\%-40\%) cells (53). Therefore, BTK inhibition may reduce the frequency of B10Pro-like CLL cells via 2 mutually nonexclusive mechanisms: by directly inhibiting the IL-10 production in CLL cells, and/or by selectively depleting B10Prolike CLL cells. IL-10 is a major immunosuppressive cytokine that can be produced by multiple cell types. Surprisingly, it has been found that B cells are actually a dominant source of IL-10 in vivo in both naive and immune system-activated mice (54). Secretion of IL-10 by CLL cells could be triggered by infections or host inflammatory responses in CLL. Given the significantly elevated number of malignant B cells in CLL patients, production of IL-10 by even a small fraction of the tumor cells could cause significant immune suppression (5). Endogenous B10/Breg cells were shown to inhibit $\mathrm{CD} 20 \mathrm{mAb}$-induced lymphoma regression by secreting IL-10 and inhibiting mAb-mediated monocyte activation (55). CLL cell IL-10 production was also found to significantly inhibit monocyte activation (5). Therefore, by reducing the IL-10 production by CLL cells, ibrutinib treatment could lead to relief of CLL-induced immune suppression. Moreover, as B10/B10Pro cells share many phenotypic and functional features with CLL cells, it is possible that ibrutinib treatment also depletes these immunosuppressive cells or impairs their function.

Our study includes comparative data from CLL patient samples obtained at matched time points during treatments with either an irreversible ITK/BTK inhibitor or more selective BTK inhibitor. Although descriptive, these experiments identify effects such as expansion of effector $\mathrm{T}$ cells, increased proportion of Th17producing cells, and distinct changes in CTLA-4 intracellular expression between $\mathrm{CD} 4^{+}$and $\mathrm{CD} 8^{+}$subsets that are likely attributable to alternative, non-BTK targets such as ITK that are inhibited by ibrutinib but not acalabrutinib. We also provide in vitro evidence that ibrutinib but not acalabrutinib prevents AICD of activated T cells and NK cells. In contrast, decreased expression of surface PD- 1 on $\mathrm{CD}^{+}$and $\mathrm{CD} 8^{+} \mathrm{T}$ cells and intracellular CTLA-4 on $\mathrm{CD}^{+} \mathrm{T}$ cells was observed with both agents, implicating BTK as an indirect factor in this change. This may be through modulation of immune-suppressive molecules (CD200, IL-10, and others) on CLL tumor cells, which are impacted by both agents, and further studies to understand these effects are ongoing. Due to the smaller sample size for acalabrutinib-treated patients available for analysis, some of the negative findings on acalabrutinib were underpowered. For example, there is also a trend towards a decrease in the percentage of Treg cells after acalabrutinib treatment, and with increased sample size, this could be a statistically significant difference - albeit more modest compared with the difference observed in ibrutinib-treated samples. Nonetheless, our data presented here identify ibrutinib and acalabrutinib as distinctly different immunemodulating agents. Despite potential added toxicity with ibrutinib due to alternative target inhibition, our data show improved immune modulation in vivo with this agent compared with acalabrutinib and support its use in combination with other immune therapies.

In summary, by studying CLL patients treated with ibrutinib, we have identified its superior immunomodulatory effects by virtue of being a less specific inhibitor of BTK. Ibrutinib induces significant increases in $\mathrm{T}$ cell numbers that are not achieved by a more selective BTK inhibitor. The underlying mechanism is likely to be ITK inhibition that leads to the rescue of chronically stimulated $\mathrm{T}$ cells from AICD. Our data therefore provide support for ibrutinib therapy as an ideal cellular immune-modulating agent for CLL and potentially other types of hematologic and solid cancers. For example, ibrutinib can be incorporated as part of cellular immune therapy. In vivo persistence and expansion of antigen-specific $\mathrm{T}$ cells is the most critical determining factor for the success of adoptive immunotherapy with tumor-infiltrating lymphocytes (TILs) and chimeric antigen receptor (CAR) T cells (56). Expanding such cells with systemic administration of IL-2 is toxic and may have deleterious effect on the long-term persistence of antitumor immunity (46). IL-2 also leads to preferential expansion of Treg cells (57). Preconditioning with lymphocyte depletion enhances homeostatic proliferation and depletes host Treg cells. However, it also carries along significant 
A Pretreatment

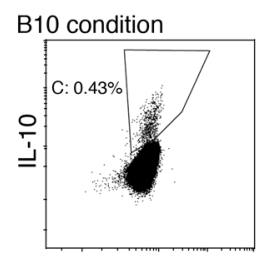

Cycle 3 day 1

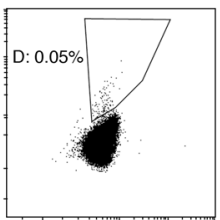

B10Pro condition

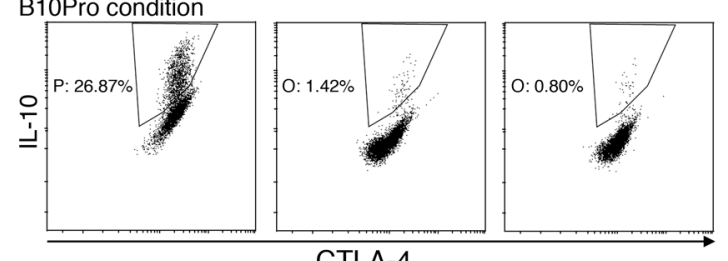

CTLA-4

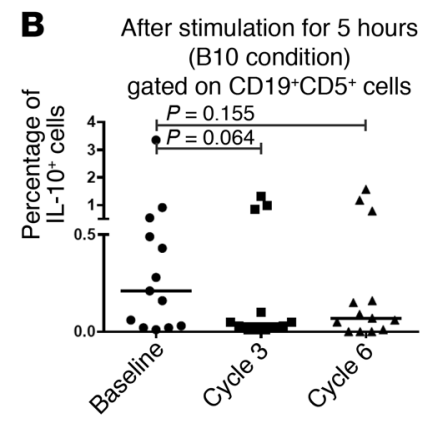

After stimulation for 48 hours (B10Pro condition) gated on $\mathrm{CD} 19+\mathrm{CD} 5+$ cells
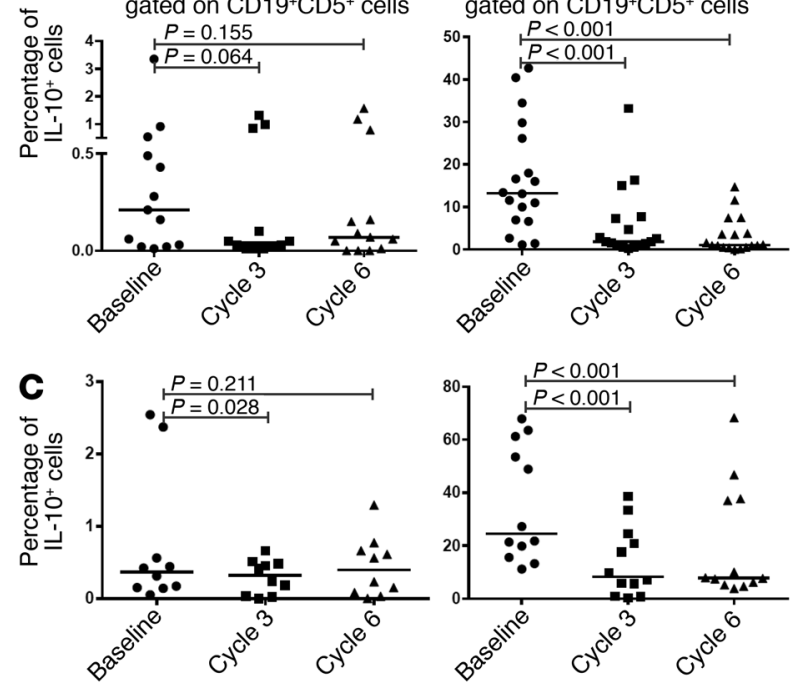

Figure 8. Ability of CLL cells to produce IL-10 is impaired with BTK inhibitor treatment. PBMCs from CLL patients were collected before and during treatment with ibrutinib $(\mathbf{A}-\mathbf{C})$ and acalabrutinib (D). Cells were stimulated in vitro with $\mathrm{CpG}$ and $\mathrm{PMA}$ /ionomycin for 5 hours (B10 conditions) or with $\mathrm{CpG} / \mathrm{CD} 40 \mathrm{~L}$ for 48 hours, with PMA/ionomycin added for the last 5 hours (B10Pro conditions). IL-10 production was detected by intracellular cytokine staining. All events were gated on CLL cells (CD19+CD5+CD3-). (A) Representative flow cytometry plots of IL-10 expression in CLL cells under B10 conditions (top) and B10Pro conditions (bottom). (B) CLL samples were collected at baseline and at the beginning of cycles 3 and 6 ( 8 and 20 weeks, respectively) after starting ibrutinib treatment. Graphs show the percentages of IL-10 producing CLL cells after in vitro incubation under B10 conditions (left; $n=13$ ) and B10Pro conditions (right; $n=18$ ). (C) PBMCs were collected from CLL patients at baseline and at the beginning of cycles 3 and 6 ( 8 and 20 weeks, respectively) after starting acalabrutinib treatment. Graphs show the percentages of IL-10-producing CLL cells after in vitro incubation under B10 conditions (left; $n=10$ ) and B10Pro conditions (right; $n=12$ ). Differences were assessed using linear mixed-effects models.

toxicities, and Treg cells can out-proliferate conventional $\mathrm{T}$ cells in the lymphopenic environment (58). Low persistence of infused $\mathrm{T}$ cells may also be a result of $\mathrm{T}$ cell exhaustion (46), and ongoing clinical trials are investigating immune checkpoint blockade to boost the persistence of tumor-specific T cells. Of note, checkpoint blockade of CTLA-4 has been found to expand functional Treg cells (59). Ibrutinib enhances persistence/expansion of activated T cells and shows the following desirable qualities: (a) it has no deleterious effects on the T-CM or naive T cells, (b) it does not cause collateral expansion of the Treg cells, and (c) it partially reverses the exhausted T cell phenotype by reducing the expression of PD-1 and CTLA-4. Together, these findings provide further rationale for use of ibrutinib as an adjuvant for expansion of TILs or CAR-T cells, as our recent data demonstrated (23).

\section{Methods}

Detailed protocols are provided in the supplemental methods.

Patient samples. All patients provided written informed consent to provide material as part of an IRB-approved trial. Blood samples were collected before treatment and at completion of 2 months (cycle 3 day 1) and 5 months (cycle 6 day 1) of ibrutinib or acalabrutinib treatment from CLL patients enrolled in our blood collection study OSU-0025 that allows serial collection of samples at a monthly interval in leukemia patients. Samples described here are from NCT01589302 and NCT02029443 (clinicaltrials.gov). PBMCs were isolated from blood samples by density gradient centrifugation using Ficoll. PBMCs were then washed twice, resuspended in freezing medium containing $10 \%$ DMSO, and were stored in a liquid $\mathrm{N}_{2}$ cryopreservation system. Patient characteristics are shown in Table 1. Details of patient information are shown in Supplemental Table 2.

Mice, cell line, and antibodies. All animal experiments were carried out under protocols approved by The Ohio State University Institutional Laboratory Animal Care and Use Committee. TCL-1-transgenic mice (on the C57BL/6 background) used for these experiments have been previously described (21). Breeding pairs were provided to our group as a gift from Carlo M. Croce (The Ohio State University). OT-1 TCRtransgenic mice were purchased from The Jackson Laboratory. C1498OVA is a murine myeloid leukemia cell line ( $\mathrm{H}-2^{\mathrm{b}}, \mathrm{C} 57 \mathrm{BL} / 6$ background) expressing the experimental surrogate antigen ovalbumin. It was provided by Bruce R. Blazar (University of Minnesota, Minneapolis, Minnesota, USA). All antibodies used are listed in Supplemental Table 3.

Statistics. Separate linear mixed-effects models were used to assess differences (cycles 3 and 6 vs. baseline) in cell number and percentage as well as mean fluorescence intensity (MFI) among the different subsets for patients treated with ibrutinib and acalabrutinib. Absolute cell number and MFI data were first log-transformed to reduce skewness and stabilize variances. An initial analysis was performed based on 17 patients treated with ibrutinib and 9 patients treated with acalabrutinib. Later, an additional 2 patients with ibrutinib and 4 patients with acalabrutinib were added to the original cohorts and the analysis was repeated. Results are shown for the updated data with a total of 19 ibrutinib patients and 13 acalabrutinib patients. A comparison of the original and updated results is provided in Supplemental Table 4. Not all experiments were performed on each patient's serial sample; therefore, the actual $n$ for each experiment was less than 19 and 13 for ibrutinib- and acalabrutinib-treated patients, respectively. All clinical-sample analyses were performed using SAS/STAT software, version 9.4 of the SAS System for Windows (SAS Institute Inc.). For the in vitro and animal experiments described Supplemental Figures 2, 7, and 8, a 2-tailed Student's $t$ test was used. A $P$ value less than 0.05 was considered significant for all the experiments.

Study approval. The studies on human samples were reviewed and approved by The Ohio State University IRBs. All subjects provided appro- 
priate prior informed consent to participate in the study. The respective studies on C57BL/6 WT, TCL-1-transgenic, and OT-1-transgenic animals were reviewed and approved by the IACUC at The Ohio State University.

\section{Author contributions}

ML contributed to the designing and performing of the experiments, data analysis, and writing the manuscript. KB, PD, CC, and AG helped sample processing and performing of the experiments. BLM and MAC designed and performed the NK cell ADCC experiments. AML performed the statistical analysis and interpretation of the data. KJM, JAJ, JMF, LAA, FA, and JAW contributed to provision of study materials/recruiting of patients. JAF, CHJ, MVM, MAC, AJJ, and NM critically reviewed the manuscript and helped with the interpretation of the data. JCB and NM designed the study, secured funding, directed the research, analyzed data, and had overall oversight of the trial and manuscript.

\section{Acknowledgments}

JAF acknowledges funding from the University of Pennsylvania during this study. This work was supported by the National Cancer Institute (NCI): T32CA009140 (JAF), K08166039 (MVM), P01 CA095426 (AJ, NM, JCB), K23 CA178183 (JAW), R35 CA198183 (JCB), R01 CA 177292 (JCB, AJJ), R01 CA197870 (JAW, AJJ), 5 K12 CA133250-08 (ML) and P30 CA016058 (MAC, JCB), as well as Michael and Judy Thomas, the D. Warren Brown Foundation, and the Four Winds Foundation.

Address correspondence to: John C. Byrd or Natarajan Muthusamy, The Ohio State University Comprehensive Cancer Center, 455 Wiseman Hall, 400 West 12th Avenue, Columbus, Ohio 43210, USA. Phone: 614.293.8330; Email: john.byrd@osumc.edu (J.C. Byrd); Phone: 614.685.2407; Email: raj.muthusamy@osumc. edu (N. Muthusamy).
1. Riches JC, Gribben JG. Understanding the immunodeficiency in chronic lymphocytic leukemia: potential clinical implications. Hematol Oncol Clin North Am. 2013;27(2):207-235.

2. Brusa D, et al. The PD-1/PD-L1 axis contributes to T-cell dysfunction in chronic lymphocytic leukemia. Haematologica. 2013;98(6):953-963.

3. Attia MA, Nosair NA, Gawally A, Elnagar G, Elshafey EM. HLA-G expression as a prognostic indicator in B-cell chronic lymphocytic leukemia. Acta Haematol. 2014;132(1):53-58.

4. Pallasch CP, Ulbrich S, Brinker R, Hallek M, Uger RA, Wendtner CM. Disruption of T cell suppression in chronic lymphocytic leukemia by CD200 blockade. Leuk Res. 2009;33(3):460-464.

5. DiLillo DJ, et al. Chronic lymphocytic leukemia and regulatory B cells share IL-10 competence and immunosuppressive function. Leukemia . 2013;27(1):170-182.

6. Gorczynski RM, Lee L, Boudakov I. Augmented induction of $\mathrm{CD} 4{ }^{+} \mathrm{CD} 25^{+}$Treg using monoclonal antibodies to CD200R. Transplantation. 2005;79(9):1180-1183.

7. Memarian A, et al. Upregulation of CD200 is associated with Foxp $3^{+}$regulatory $\mathrm{T}$ cell expansion and disease progression in acute myeloid leukemia. Tumour Biol. 2013;34(1):531-542.

8. Jitschin R, et al. CLL-cells induce IDO ${ }^{\text {hi }}$ CD14 ${ }^{+}$HLA $^{-D R^{\text {lo }}}$ myeloid-derived suppressor cells that inhibit $\mathrm{T}$-cell responses and promote TRegs. Blood. 2014;124(5):750-760.

9. Riches JC, et al. T cells from CLL patients exhibit features of T-cell exhaustion but retain capacity for cytokine production. Blood. 2013;121(9):1612-1621.

10. Forconi F, Moss P. Perturbation of the normal immune system in patients with CLL. Blood. 2015;126(5):573-581.

11. Podhorecka M, Dmoszynska A, Rolinski J, Wasik E. T type 1 /type 2 subsets balance in B-cell chronic lymphocytic leukemia--the three-color flow cytometry analysis. Leuk Res. 2002;26(7):657-660.

12. Jadidi-Niaragh F, et al. Downregulation of IL-17-producing $\mathrm{T}$ cells is associated with regulatory $\mathrm{T}$ cell expansion and disease progression in chronic lymphocytic leukemia. Tumour Biol. 2013;34(2):929-940.
13. Hus I, et al. Th17/IL-17A might play a protective role in chronic lymphocytic leukemia immunity. PLOS ONE. 2013;8(11):e78091.

14. Tang D, Niu Q, Jiang N, Li J, Zheng Q, Jia Y. Increased frequencies of Th17 in the peripheral blood of patients with chronic lymphocytic leukemia: a one year follow-up. PakJ Med Sci. 2014;30(5):1128-1133.

15. Jain $P$, et al. Th17 and non-Th17 interleukin-17expressing cells in chronic lymphocytic leukemia: delineation, distribution, and clinical relevance. Haematologica. 2012;97(4):599-607.

16. Strati $P$, et al. Lenalidomide induces long-lasting responses in elderly patients with chronic lymphocytic leukemia. Blood. 2013;122(5):734-737.

17. Sagiv-Barfi I, Kohrt HE, Czerwinski DK, Ng PP, Chang BY, Levy R. Therapeutic antitumor immunity by checkpoint blockade is enhanced by ibrutinib, an inhibitor of both BTK and ITK. Proc Natl Acad Sci U S A. 2015;112(9):E966-E972.

18. Sagiv-Barfi I, Kohrt HE, Burckhardt L, Czerwinski DK, Levy R. Ibrutinib enhances the antitumor immune response induced by intratumoral injection of a TLR9 ligand in mouse lymphoma. Blood. 2015;125(13):2079-2086.

19. Kondo K, et al. Ibrutinib can modulate the $\mathrm{T}$ cell response in chronic lymphocytic leukemia by reducing PD1/PDL1 interactions. Blood. 2015;126(23):1737.

20. Dubovsky JA, et al. Ibrutinib treatment ameliorates murine chronic graft-versus-host disease. J Clin Invest. 2014;124(11):4867-4876.

21. Dubovsky JA, et al. Ibrutinib is an irreversible molecular inhibitor of ITK driving a Th1selective pressure in T lymphocytes. Blood. 2013;122(15):2539-2549.

22. Gunderson AJ, et al. Bruton tyrosine kinasedependent immune cell cross-talk drives pancreas cancer. Cancer Discov. 2016;6(3):270-285.

23. Fraietta JA, et al. Ibrutinib enhances chimeric antigen receptor T-cell engraftment and efficacy in leukemia. Blood. 2016;127(9):1117-1127.

24. Ruella M, et al. The addition of the BTK inhibitor ibrutinib to anti-CD19 chimeric antigen receptor T cells (CART19) improves responses against mantle cell lymphoma. Clin Cancer Res. 2016;22(11):2684-2696.
25. Byrd JC, et al. Acalabrutinib (ACP-196) in relapsed chronic lymphocytic leukemia. $N$ Engl J Med. 2016;374(4):323-332.

26. Sallusto F, Geginat J, Lanzavecchia A. Central memory and effector memory $\mathrm{T}$ cell subsets: function, generation, and maintenance. Annu Rev Immunol. 2004;22:745-763.

27. Ponader S, et al. The Bruton tyrosine kinase inhibitor PCI-32765 thwarts chronic lymphocytic leukemia cell survival and tissue homing in vitro and in vivo. Blood. 2012;119(5):1182-1189.

28. Miller AT, Berg LJ. Defective Fas ligand expression and activation-induced cell death in the absence of IL-2-inducible T cell kinase. J Immunol. 2002;168(5):2163-2172.

29. Sun Y, et al. Inhibition of the kinase ITK in a mouse model of asthma reduces cell death and fails to inhibit the inflammatory response. Sci Signal. 2015;8(405):ra122.

30. Green DR, Droin N, Pinkoski M. Activationinduced cell death in T cells. Immunol Rev. 2003;193:70-81.

31. Ross ME, Caligiuri MA. Cytokine-induced apoptosis of human natural killer cells identifies a novel mechanism to regulate the innate immune response. Blood. 1997;89(3):910-918.

32. Khurana D, Arneson LN, Schoon RA, Dick CJ, Leibson PJ. Differential regulation of human NK cell-mediated cytotoxicity by the tyrosine kinase Itk. J Immunol. 2007;178(6):3575-3582.

33. Bao Y, et al. Tyrosine kinase Btk is required for NK cell activation. J Biol Chem. 2012;287(28):23769-23778.

34. Gattinoni L, et al. A human memory T cell subset with stem cell-like properties. Nat Med. 2011;17(10):1290-1297.

35. Zhu J, Yamane H, Paul WE. Differentiation of effector CD4 T cell populations $\left(^{*}\right)$. Annu Rev Immunol. 2010;28:445-489.

36. Gomez-Rodriguez J, et al. Itk-mediated integration of $\mathrm{T}$ cell receptor and cytokine signaling regulates the balance between Th17 and regulatory T cells. J Exp Med. 2014;211(3):529-543.

37. Tedder TF. B10 cells: a functionally defined regulatory B cell subset. JImmunol. 2015;194(4):1395-1401.

38. Brunner C, Avots A, Kreth HW, Serfling E, Schus- 


\section{CLINICAL MEDICINE}

ter V. Bruton's tyrosine kinase is activated upon CD40 stimulation in human B lymphocytes. Immunobiology. 2002;206(4):432-440.

39. Haxhinasto SA, Bishop GA. Synergistic B cell activation by $\mathrm{CD} 40$ and the $\mathrm{B}$ cell antigen receptor: role of B lymphocyte antigen receptormediated kinase activation and tumor necrosis factor receptor-associated factor regulation. J Biol Chem. 2004;279(4):2575-2582.

40. Davids MS, Brown JR. Ibrutinib: a first in class covalent inhibitor of Bruton's tyrosine kinase. Future Oncol. 2014;10(6):957-967.

41. Liao XC, Fournier S, Killeen N, Weiss A, Allison JP, Littman DR. Itk negatively regulates induction of $\mathrm{T}$ cell proliferation by CD28 costimulation. J Exp Med.1997;186(2):221-228.

42. Lin TA, et al. Selective Itk inhibitors block T-cell activation and murine lung inflammation. Biochemistry. 2004;43(34):11056-11062.

43. Niemann CU, et al. Disruption of in vivo chronic lymphocytic leukemia tumor-microenvironment interactions by ibrutinib--findings from an investigator-initiated phase II study. Clin Cancer Res. 2016;22(7):1572-1582.

44. Zenz T. Exhausting T cells in CLL. Blood. 2013;121(9):1485-1486.

45. Pakker NG, et al. Biphasic kinetics of peripheral blood $\mathrm{T}$ cells after triple combination therapy in
HIV-1 infection: a composite of redistribution and proliferation. Nat Med.1998;4(2):208-214

46. Kalos M, June CH. Adoptive T cell transfer for cancer immunotherapy in the era of synthetic biology. Immunity. 2013;39(1):49-60.

47. Natarajan G, et al. Ibrutinib enhances IL-17 response by modulating the function of bone marrow derived dendritic cells. Oncoimmunology. 2016;5(1):e1057385.

48. Zhang Y, Xu G, Zhang L, Roberts AI, Shi Y. Th17 cells undergo Fas-mediated activation-induced cell death independent of IFN-gamma. J Immunol. 2008;181(1):190-196.

49. Coles SJ, et al. Increased CD200 expression in acute myeloid leukemia is linked with an increased frequency of $\mathrm{FoxP}^{+}$regulatory $\mathrm{T}$ cells. Leukemia. 2012;26(9):2146-2148.

50. Schmidt NW, Thieu VT, Mann BA, Ahyi AN, Kaplan MH. Bruton's tyrosine kinase is required for TLR-induced IL-10 production. JImmunol. 2006;177(10):7203-7210.

51. Kenny EF, Quinn SR, Doyle SL, Vink PM, van Eenennaam H, O'Neill LA. Bruton's tyrosine kinase mediates the synergistic signalling between TLR9 and the B cell receptor by regulating calcium and calmodulin. PLOS ONE. 2013;8(8):e74103.

52. Chen SS, et al. BTK inhibition results in impaired
The Journal of Clinical Investigation

CXCR4 chemokine receptor surface expression, signaling and function in chronic lymphocytic leukemia. Leukemia. 2016;30(4):833-843.

53. Khan WN, et al. Defective B cell development and function in Btk-deficient mice. Immunity. 1995;3(3):283-299.

54. Madan R, et al. Nonredundant roles for B cellderived IL-10 in immune counter-regulation. JImmunol. 2009;183(4):2312-2320.

55. Horikawa M, Minard-Colin V, Matsushita T, Tedder TF. Regulatory B cell production of IL-10 inhibits lymphoma depletion during CD20 immunotherapy in mice. JClin Invest. 2011;121(11):4268-4280.

56. Robbins PF, et al. Cutting edge: persistence of transferred lymphocyte clonotypes correlates with cancer regression in patients receiving cell transfer therapy. JImmunol. 2004;173(12):7125-7130.

57. Maus MV, Fraietta JA, Levine BL, Kalos M, Zhao Y, June CH. Adoptive immunotherapy for cancer or viruses. Annu Rev Immunol. 2014;32:189-225.

58. Klebanoff CA, Gattinoni L. Stubborn Tregs limit T-cell therapy. Blood. 2012;120(12):2352-2354.

59. Kavanagh B, et al. CTLA4 blockade expands FoxP $3^{+}$regulatory and activated effector $\mathrm{CD} 4^{+}$ $\mathrm{T}$ cells in a dose-dependent fashion. Blood. 2008;112(4):1175-1183. 